\title{
Cleaning of molecular machinery of cell, proteostasis, proteolysis and endocytosis selectively, effectively, and precisely: intracellular self-defense and cellular perturbations
}

\author{
Rajiv Kumar ${ }^{* 1}$, Bhupender S Chhikara², Kiran Gulia ${ }^{3}$, and Mitrabasu Chillar ${ }^{4}$ \\ ${ }^{*}$ NIET, National Institute of Medical Science, India. \\ ${ }^{2}$ Department of Chemistry, Aditi Mahavidyalaya, University of Delhi. Delhi, 110027, India. \\ ${ }^{3}$ Materials and Manufacturing, School of Engineering, University of Wolverhampton, England, UK TF29NN. \\ ${ }^{4}$ Institute of Nuclear Medicine and Allied Sciences (INMAS) Brig. S. K. Mazumdar Marg, Delhi, 110054 \\ E-mail address of the corresponding author: Dr. Rajiv Kumar, chemistry_rajiv@hotmail.com
}

The authors dedicate this review article to Hon'ble Dr. Yoganand Shastri Ji, Distinguished Professor, University of Delhi; Honorable Speaker of Delhi Legislative Assembly; Cabinet Minister for Development, Food and Civil Supplies; and Cabinet Minister for Health and Social Welfare (Delhi Legislative Assembly).

\begin{abstract}
Network coordinates of cellular processes (proteostasis, proteolysis, endocytosis), and molecular chaperones are the key complements in cell machinery, processes. Specifically cellular pathways are answerable for conformational maintenance, cellular concentration, interactions, protein synthesis, disposal of misfolded proteins, localization, folding, and degradation. Failures in cellular processes and pathways disturb structural proteins and nucleation of amyloid. These mishappenings further initiate amyloid polymorphism, transmissibility, co-aggregation of pathogenic proteins in tissues and cells, prion strains, mechanisms and pathways of toxicity. These conditions favor and lead the formation of elongated, fibers, and spines amyloid fibrils consisting of many-stranded $\beta$-sheets $(N$, $\mathrm{N}$-terminus; C, C-terminus), abnormal fibrous, extracellular, proteinaceous deposits. Finally, these $\beta$ sheets, deposit, thereafter, the cells fail to degrade them effectively. The essential torsion angles ( $\varphi$, $\psi$, and $\omega$ ) define the protein conformation and their architecture as well. Cells initiate several transformations and pathways during the regulation of the protein homeostasis as per the need of the cell functioning which were governed by ATP-dependent proteases. In the process, the kinetic of molding/folding phenomenon is disturbed, and later on, it get dominated by cross-domain misfolding intermediates, but, simultaneously, it gets opposed by small stretching forces that naturally exist in the cell. The Ubiquitin/proteasome system deals with the damaged proteins which are not refolded by chaperone types of machinery. Ubiquitin-protein ligases (E3-Ub) participated in all the cellular reactivity initiated and governed by the molecular chaperones to stabilize the cellular proteome and participate in the degradation phenomenon adopted for the damaged proteins. The
\end{abstract}


optical tweezers, single resolution based technique, disclose the folding pathway of linear chain protein, how did they convert themselves into three-dimensional architecture. Further, DNA-protein conjugation analysis determines to extract folding energies as single-molecule kinetic and thermodynamic statistics.

\section{Graphical abstract}

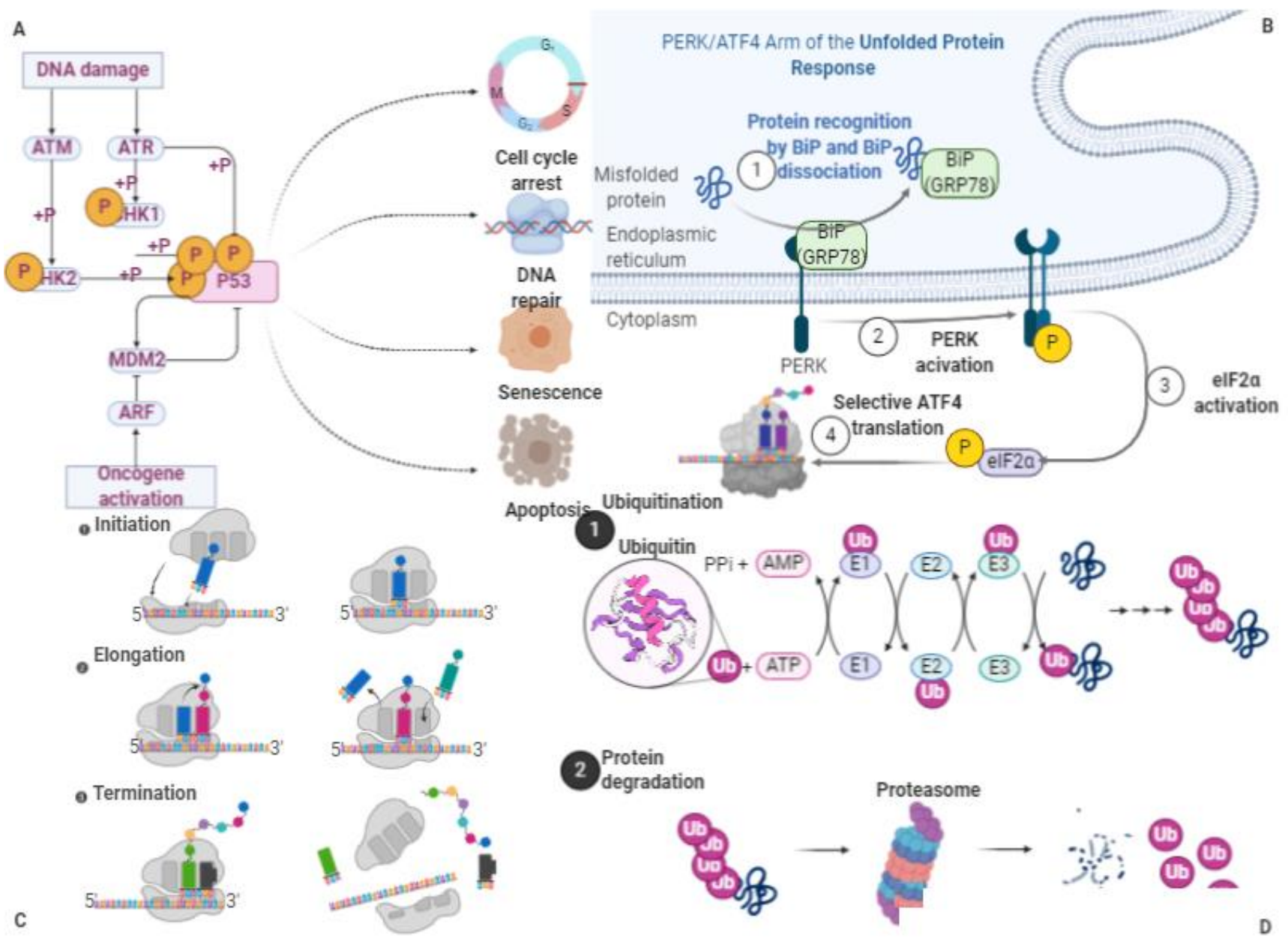

Illustrations, in four parts, of the regulation of the ubiquitin-protein ligases and the ubiquitylation, a dynamic cellular process for stability, and protein folding induced. The ubiquitin-signaling tense the ubiquitin-conjugation machinery for accurate surveillance, cell cycle arrest, DNA damage and repair, senescence, and apoptosis. Unfolded protein response, dissociation and protein recognitions. Processes: initiation, elongation and termination. "Adapted and created [biorender.com]. Same is referred and acknowledged as per instructions".

\section{Introduction}

Cells are always capable to control and hold the equilibrium of numerous pathways (folding, biochemical transformation, synthesis mechanism, and degradation of misfolded proteins) necessary for sustainability and proper functioning. Among them, the protein misfolding and aggregation is not the natural processes, as these are caused by irregular, unnatural, unwanted environmental factors. 
There is a growing list of diseases triggered by protein misfolding and aggregation. ${ }^{1}$ Some mechanisms (cytoplasmic quality control, molecular chaperones, and ubiquitin-proteasome system) assist with protein folding and open the door for the deterioration of misfolded polypeptides. ${ }^{2}$ Genetic mutations, translational faults, irregular protein alterations, thermal or oxidative stress, and imperfect complex growths initiate protein misfolding. Therefore, this is an opportunity for pathological conditions (inappropriate degradation, dominant-negative mutations, essential modifications, mislocalization, novel toxic utilities, and amyloid buildup) to be rooted in it. ${ }^{3}$ Thus, these intricacies in the cellular environment craft foot race in the natural functioning of the cells and entrap functional proteins. ${ }^{4}$ As, normal proteins cannot achieve their correct conformations and finally, misfolding get initiated in proteins. The configurations of the proteins simultaneously adopted analogous folding and favor the materialization of the inherent configuration. It contains a hydrophobic core cluster required for the formation of the $\beta$-hairpin which is a perfect folded assembly. On the other hand, the process of the misfolding of the proteins produced amyloid fibrils which is a nonnative turn configuration. These irregular activities, disturbed the packing of hydrophobic core cluster and initiate the formation of the misfolded assembly. Competing (misfolded) structures, however, could be initiated by various cellular pathways, mechanisms, phenomenon (transcription, translation, signal transduction, genomic mutations, toxic compounds, environmental stresses, different types of cellular stress, nutrient deprivation, hostile environments, dominant-negative mutations, trafficking defects, irregular post-translational alterations, high degradation percentage, off-pathway folding, oxidative stress, reactive oxygen species, oxygen radicals, oxygenic energy transduction, Lewis acids/base regulators, oxidants, metabolism, mitochondria-to-nucleus signaling pathway, proteostasis-ubiquitin proteasomal and lysosomal autophagy systems, mitochondrial electron transport chain, cell necrosis, endothelial apoptosis, angiogenesis, and proteasomal degradation heat, and heavy metal ions) and the other specific factors. $^{5}$ These above-mentioned pathways, mechanisms, and phenomena influence the thermodynamic and kinetic parameters of the proteins.

The folding and misfolding of the proteins are then dynamically described by free energy background investigation, the output points out that the folding and misfolding of $\beta$-hairpin are analogous processes. The selection of the protein pathways to be followed in a significance of the rivalry between the construction of flexible turn conformations and cross-strand hydrophobic interface/interaction. During the endoplasmic reticulum-associated degradation (ERAD) pathway, molecular chaperones and other allied factors identify an approach, the components for the retro translocation in 
cytoplasm. ${ }^{6}$ During these processes, these proteins are tarnished by the ubiquitin-proteasome mechanism. ${ }^{7}$ There are three main sets of nonnative conformations that are stable misfolded, and unstable misfolded, and aggregation-prone respectively have extra three different consequences: functional shortage, dominant-negative possessions, or toxic cellular impacts. Protein misfolding pathologies lean to emphasis utmost towards on intra- or extra-cellular aggregation during the diseases in the proteins displaying a gain-of-functional pathology.

The $\mathrm{Ca}^{2+}$ homeostasis channeled through the ER and its higher concentration suitable for the proper folding of the proteins. Furthermore, computational studies of protein-surface interactions, ER, mitochondrial, nuclear, and ribosomal PQCPs, processes of protein folding are dynamically inflated. The setup of chaperones contributes in folding by ATP hydrolysis to identify wide-open hydrophobic amino acid spread-out layers. ${ }^{8}$ Such other pathways and mechanisms also require further investigations for a fruitful outcome. Failures of cell cleaning mechanisms for misfolded-protein within a cell is distinctively liable for various complications and diseases (protein conformational disorders, trafficking defects, mitochondria-to-nucleus signaling pathway, cardiovascular diseases, neuronal dysfunction, endothelial dysfunction, aqueous-deficient dry eyes, cataract, proteasome activity, pick's disease, and ubiquitination in the pathogenesis of human diseases, Cystic fibrosis, Dialysis-related amyloidosis, Alzheimer's disease, neurodegenerative disorders, transmissible spongiform, serpindeficiency disorders, cancer, hemolytic anemia, Huntington disease, amyotrophic lateral sclerosis, encephalopathies, Parkinson disease, and age-related molecular degeneration $\left.{ }^{9}\right) .{ }^{10}$ With the specific aim of "Cell Cleaning: Proteostasis and Proteolysis" about how to stop the cell from doing denaturing activities ${ }^{11}$ has been a point of analysis made possible by computational and theoretical advances that have perfected trials by revealing few of the folding machineries at atomic scale.

Elucidating the molecular mechanism of diseases, then design and develop the required therapies with new methodologies ${ }^{12,13}$ must be the main concern. Besides all, there are protein quality control mechanisms (cytoplasmic quality control, molecular chaperones, and ubiquitin-proteasome system) that always control protein homeostasis and provide protection during acute inflammation and stress conditions to be considered specifically. ${ }^{14}$ The failures of these mechanisms and uneven homeostasis originate from diseases and aging. Here, the authors are covering the most important cellular processes and mechanisms at the molecular, and cellular levels. Therefore, in this review article, authors are addressing the needs to find out the pathways of the perturbations in cellular proteostasis, proteolysis, and endocytosis by pointing out the urgencies for repairing and cleaning of the molecular machinery of cells selectively, effectively, and precisely. 


\section{Protein quality control pathways: molecular chaperone-mediated autophagy}

Inconsistency, dysfunctionalities, and erred in the folding of the proteins because of transcriptional, translational (Fig. 1), mutations, and oxidative stress interrupt natural cellular processes. ${ }^{15}$ Molecular chaperones notify such folded proteins, through hydrophobic patches and help them to gain their native states accordingly. If it did not happen, then proteolytic processes ${ }^{16}$ and mechanisms (ubiquitin-proteasome and autophagic vacuolar, lysosomal) can easily differentiate between folded and misfolded proteins, tailed misfolded proteins, and eradicate it, through a natural cell cleaning process.

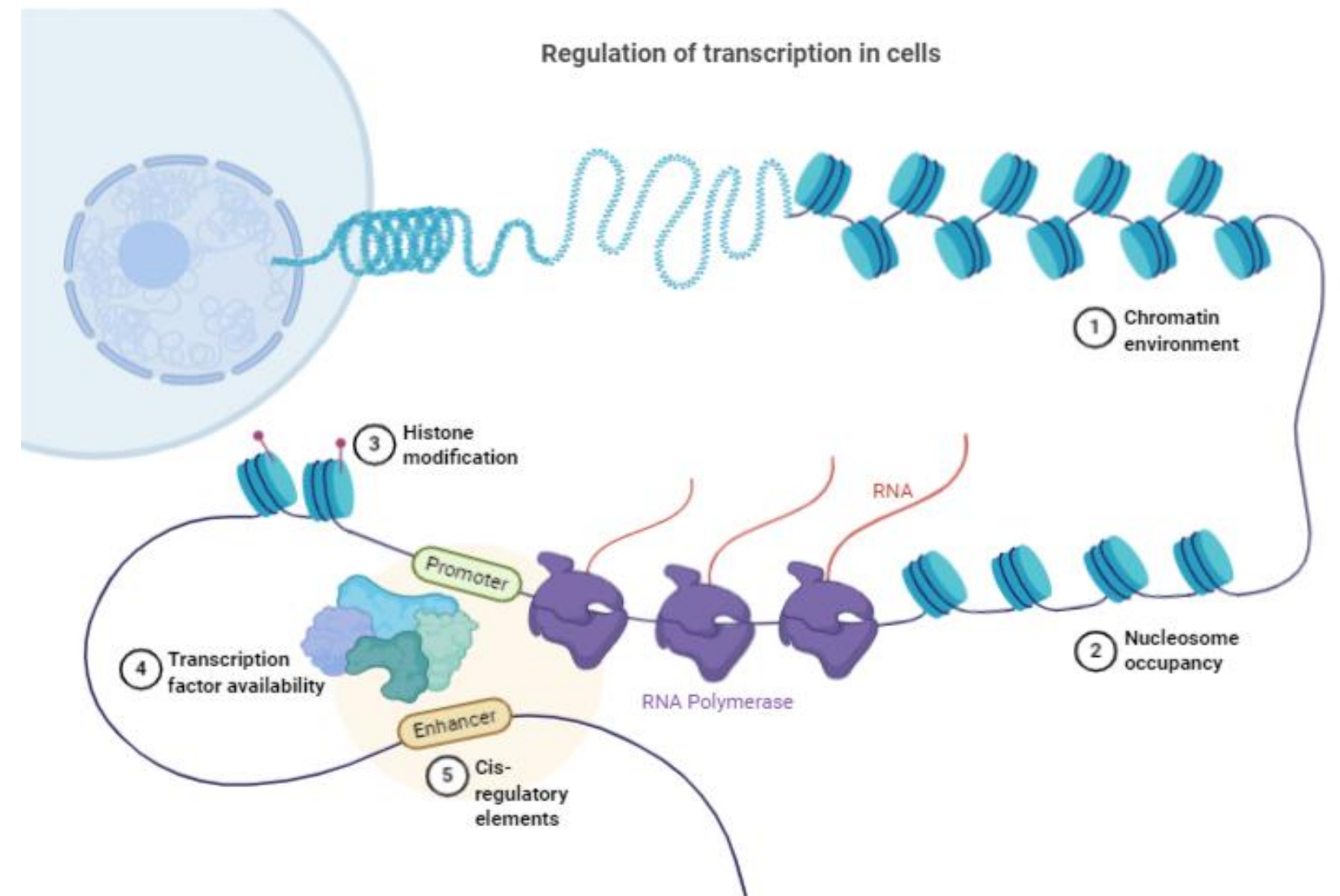

Fig. 1 Illustrations of the regulation of transcription in cells. "Adapted and created with permission from [biorender.com]. Same is referred and acknowledged as per instructions".

There is always an urgent need for quick cell cleaning via protein homeostasis (proteostasis) especially, for neuron cells that have long extensions of cellular space. If there is no proper cleaning in these cells, it will be a worse condition for cell heath. Such complications are among the main causes responsible for the pathogenesis of several diseases. In this section, the $\alpha$-Syn assemblies and autophagy-lysosome pathway were additionally included to expose their interlinked phenomenon. The key to this discussion is to explore the cellular processes to know how the degradation of $\alpha$-Syn aggregates occurs. To expose these mechanisms and happenings, the protein homeostasis, protein quality-control checkpoints, autophagy-lysosome pathway, and molecular chaperones functioning 
must be reexamined. ${ }^{17}$ At the end, the remaining misfolded proteins were abolished by Golgi quality control. Macroautophagy, one more intracellular system for bulk degradation decomposed selfcomponents and as illustrated in (Fig. 2).

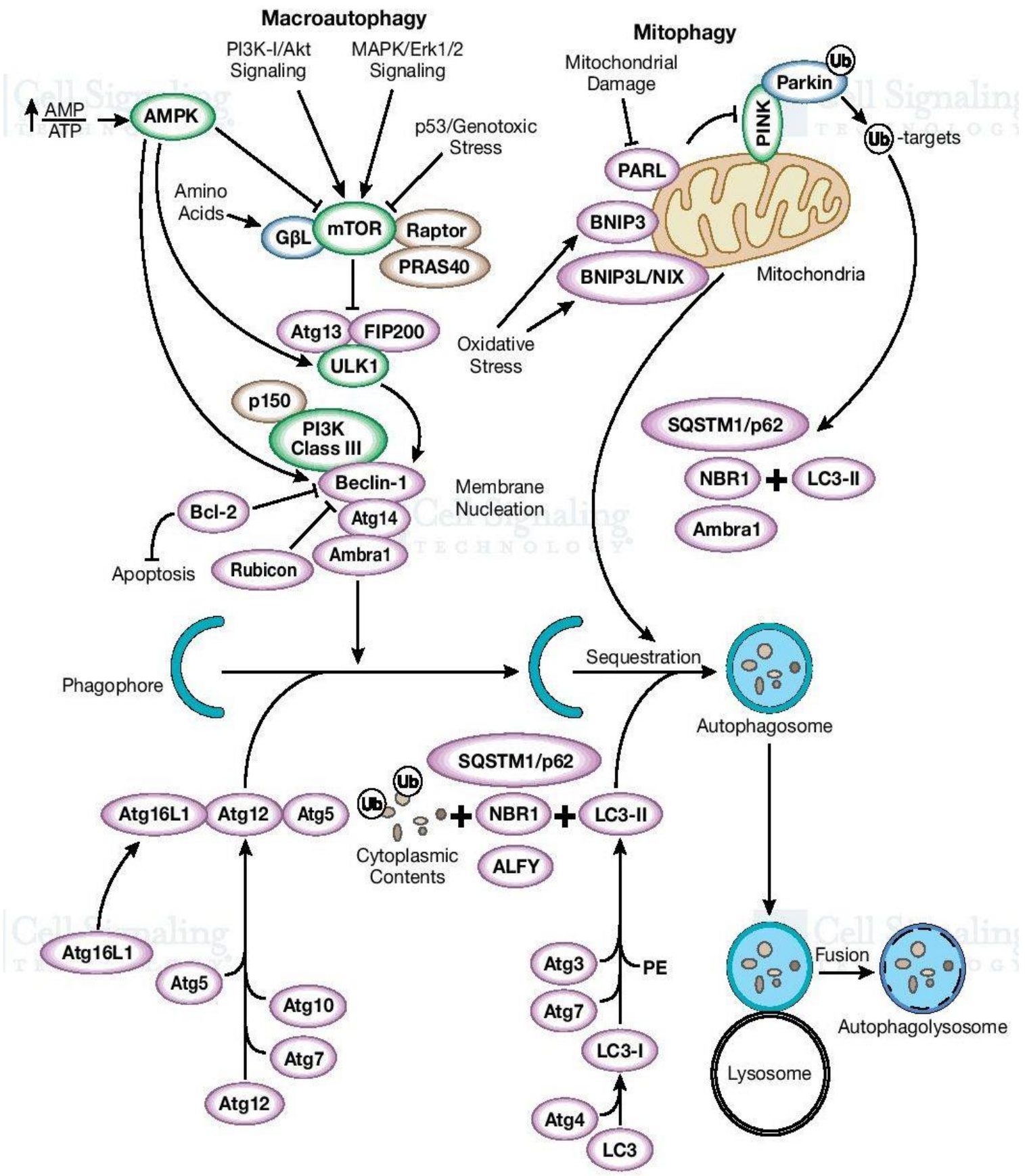

Fig. 2 Illustrations of the macroautophagy and autophagosomes-lysosomal degradation or damaged organelles. ULK1 and ULK2 formed autophagy-related (Atg) gene product (mAtg13) and the scaffold protein FIP200 (an ortholog of yeast Atg17). "Adapted with permission from [www.cellsignal.com] and the same is acknowledged.

Autophagosomes, proceed under the autophagy process to damage the cytoplasmic contents (damaged organelles, mitochondria, lysosomes, and protein aggregates) as well as invade bacteria and harmful substances, wherein proteasomal degradation pathway fails to deal with these. ${ }^{18}$ It was also highlighted in the reported literature that autophagy pathways exclusively eliminated unsafe 
substances as per the necessities of the cell, and also fulfilled the needs of the cells by providing the back-up energy and building blocks during starvation. If these harmful substances and damaged organelles remain in cell functioning, then they will play a crucial role in propagating neurodegeneration diseases. ${ }^{19}$ Therefore, underlying molecular mechanisms of autophagy redefine it as a cellular defense mechanism. Cytoplasmic, nuclear ubiquitin-proteasome system, a cytoplasmic autophagic route that can be macro, micro, or chaperone-associated autophagy, (Fig. 3) and molecular chaperones deals with the misfolded proteins always to contribute as per the necessity of cell cleaning by detecting, targeting, repairing of the damaged and mislocalized proteins existing in the cellular environment. ${ }^{20}$ To achieve a perfect methodology for convoluted cell cleaning, theoretical, experimental, and practical expertise must be involved to apply.

Knowledge of cytoplasmic protein quality control (CPQC), and ribosomal protein quality control pathways (PQCPs), must be implemented for planning new strategies to survive the failure of the protein quality control system to maintain the function and integrity of the cellular system. ${ }^{21}$ These are the main objective of the theme and with a line of action, to search for new healing methodologies for curing protein conformational disorders (PCDs). The networks of cellular proteins require continuous surveillance regularly to investigate the proper functioning of the proteostasis, and with needs to judge declines during these processes. The outcome reviving ubiquitin-proteasome system (UPs), trafficking defects, mitochondria-to-nucleus signaling pathway, neuronal dysfunction, endothelial dysfunction, and causes responsible for pathogenesis of diseases (for example in case of eyes: aqueous-deficient dry eyes diseases, age-related macular degeneration, cataract, pick's disease), will be helpful to match the demands of challenges of cell cleaning. ${ }^{22}$ Cellular toxicity too introduced by protein misfolding would also be a threat to cell fitness and viability. Molecular chaperones that act in defense, can impound irregular configured proteins to disturb the toxic interfaces. ${ }^{23}$ Health and aging play a crucial role in oxygen sensing, mediate calcium homeostasis, neurodegenerative diseases, cell growth, heme biosynthesis, and cell death. ${ }^{24}$ The mitochondrial respiratory chain impelled protons transversely onto the ion-impermeable interior membrane having a gradient $(\Delta \mu \mathrm{H})$ depended electrical potential $(\Delta \psi)$ and a concentration ratio $(\Delta \mathrm{pH})$, corresponding to the Nernst equation, that can be defined as $\Delta \mu \mathrm{H}=z \mathrm{~F} \Delta \psi+\mathrm{RT} \mathrm{Ln}[\mathrm{H}+] \mathrm{l} /[\mathrm{H}+]$ o. These sequences displayed key consequences in the $\mathrm{Ca}^{2+}$ transportation and dispersal. ${ }^{25}$ By considering the buffering capacity delivered by weak acids, and the actively respiring mitochondria supported by an electrical gradient through the internal membrane. 


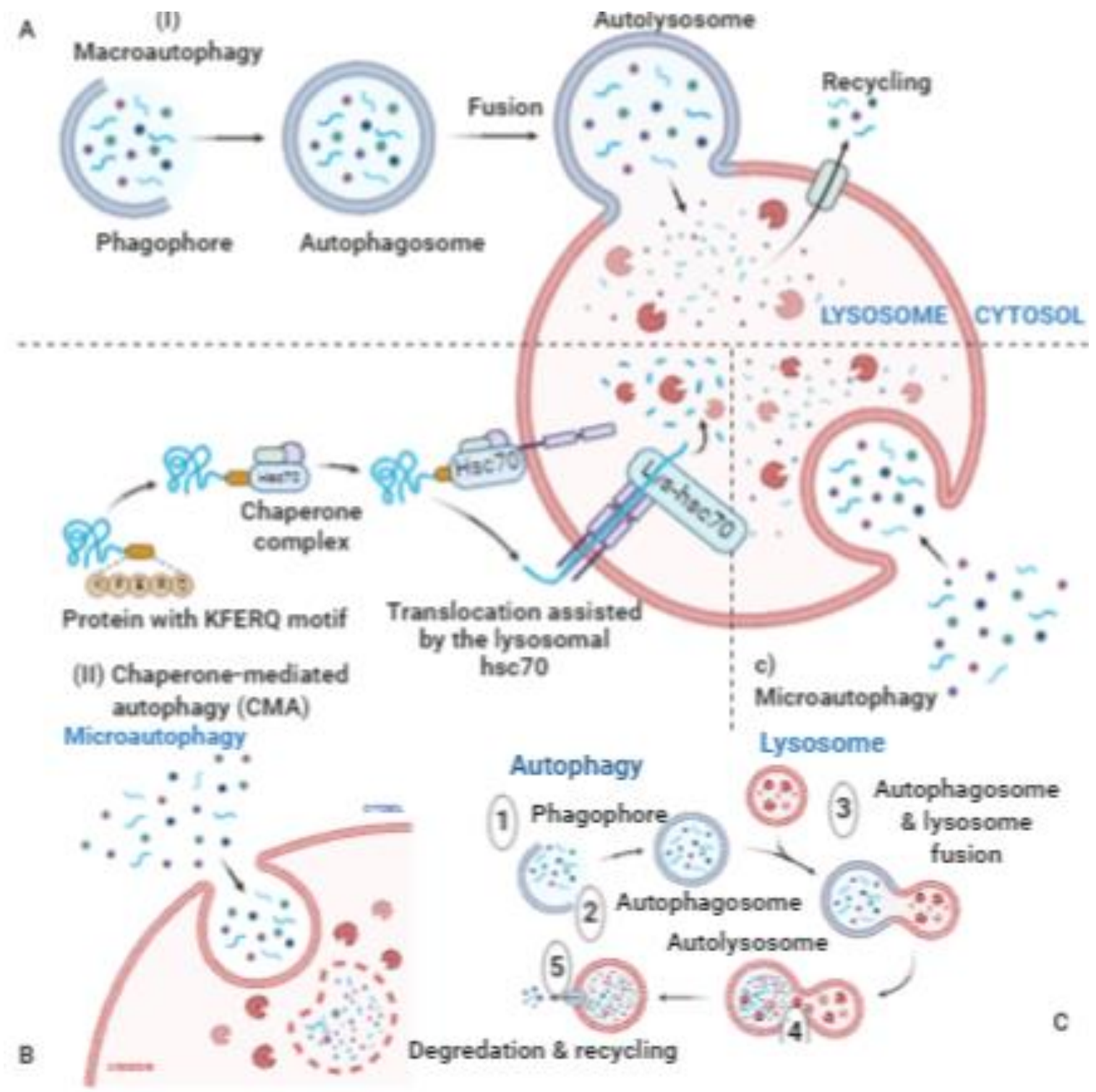

Fig. 3 Illustrations of the macroautophagy, an intracellular system for bulk degradation decomposed selfcomponents. Autophagosomes and autolysosomes and autophagy process to damage the cytoplasmic contents (damaged organelles, mitochondria, lysosomes, and protein aggregates), chaperone-mediated autophagy, microautophagy, phagophore, autophagy, degradation, and recycling. "Adapted and created with permission from [biorender.com]. Same is referred and acknowledged as per instructions".

This infers a tough thermodynamic dynamism in favor of the buildup the cations. The $\mathrm{Ca}^{2+}$ mitochondrial circulation bypassed internal mitochondrial membrane and occurred by the extrusion of $\mathrm{Ca}^{2+}$ through two major routes, one route is driven by the $\mathrm{Na}^{+} / \mathrm{Ca}^{2+}$ exchange, and another one involved via $\mathrm{H}^{+}-\mathrm{Ca}^{2+}$ exchange. In the matrix, $\mathrm{Ca}^{2+}$ excites the movement of three $\mathrm{Ca}^{2+}$ sensitive dehydrogenases in the pathway of the Krebs cycle. The gradient bypassed the internal membrane that is crucial for the mitochondrial state. This sequence occurred and influenced through the movement of the mitochondrial respiratory chain. ${ }^{26}$ Analysis of the protein conformational disorders and potentially identified protein clearance mechanisms (autophagy-lysosome, or autophagic- 
lysosomal), exposed the necessity of healthier cellular processes to achieve an extended lifespan and protected the organisms from the associated disorders, and age-associated diseases (Fig. 4). ${ }^{27}$

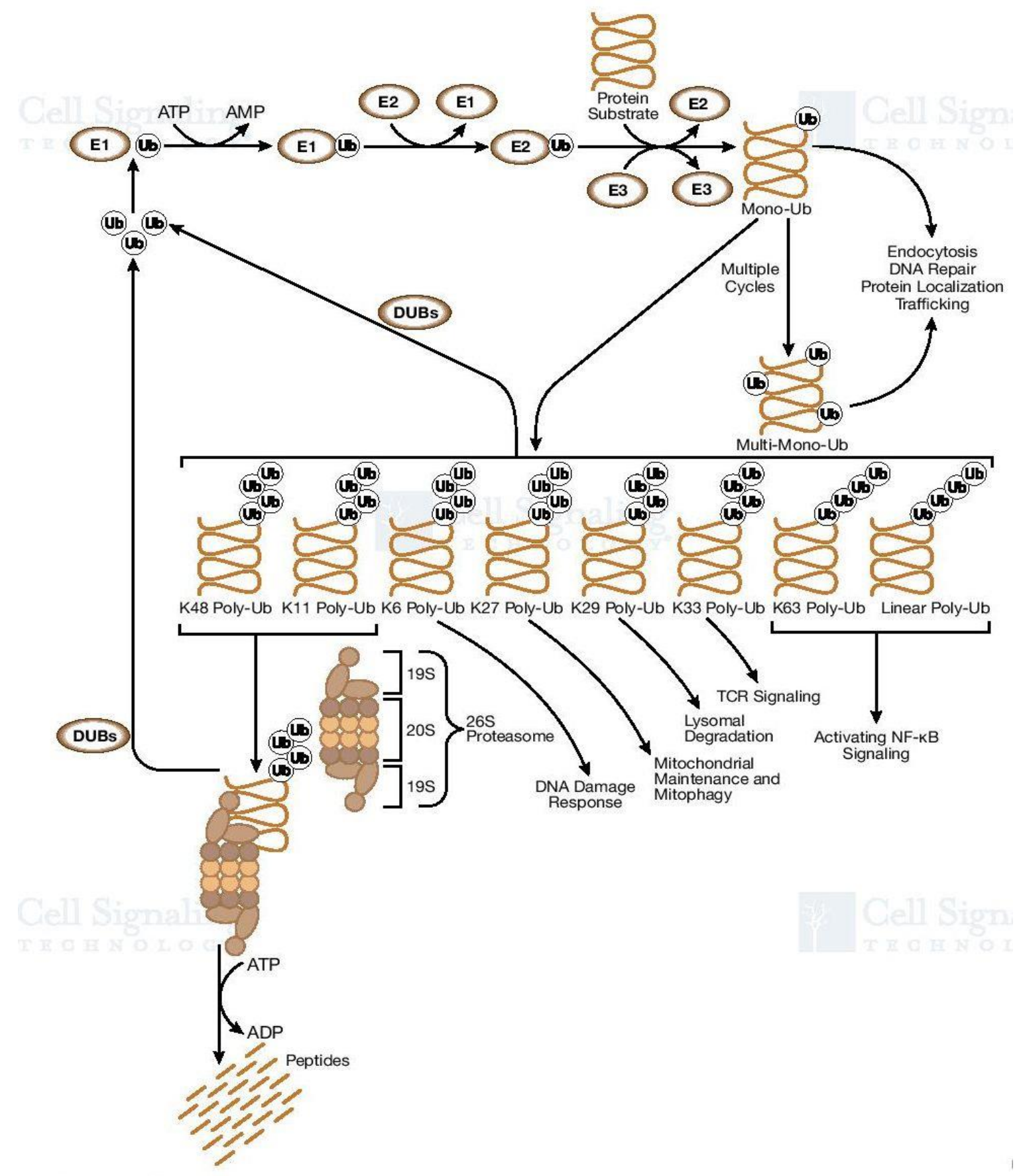

Fig. 4 Illustrations of the theoretical, experimental, practical expertization, and knowledge of cytoplasmic protein quality control (CPQC), ribosomal protein quality control pathways (PQCPs). New strategies, the failure of the protein quality control system and maintain the function and integrity of the cellular system. "Adapted with permission from [www.cellsignal.com] and the same is acknowledged.

To achieve the proper cell cleaning processes and pathways, some unknown mechanisms and hidden pathways were underlined (1) Metal-protein interface/interaction comprise metal-, ligand-, and 
enzyme-bridge complexes, define as Lewis acids/base regulators (2) Principal types of machinery maintaining proteostasis-ubiquitin proteasomal and lysosomal autophagy systems (3) Cell survival responses to environmental, stresses, oxidants, metabolism, and signal transduction (4) How do the cells adapt proteasome assembly under stress conditions, (5) Boosting cellular resilience to protein misfolding, and ER governed $\mathrm{Ca}^{2+}$ homeostasis, (6) Identifying strategies for proteasome activation by natural or chemical compounds, (7) Endothelial apoptosis, angiogenesis, and proteasomal degradation, (8) Serine-protease inhibitors, and (9) Ring-finger domain, with a dream to tailor path making strategies to dismantle unassailable fortress of the cellular system, required solemn and pierce efforts to accomplish.

\section{Dihedral angles $(\phi, \Psi)$ v's torsion angles $(\omega)$ : conformational analysis}

Protein disorders, and the conformational scattering are interlinked, thus the quantitative, and experimental analysis can evaluate the impact of these conformational changes. Further, the computational analysis and bioinformatic evidence may attest to it. This scientific evidence could be utilized to determine unfolded conditions of peptides and proteins. For the quantitative analysis of intrinsic ailment of proteins, the structure configuration and mainstay conformational entropy are highly important features. Such analysis can easily differentiate globular and disordered proteins. In the backbone torsions ( $\varphi$ and $\psi$ ) of the three-dimensional proteins, if any, minor inaccuracies originate in it which can be predicted only by computational analysis, ensued major errors. ${ }^{28}$ The dihedral angles ( $\varphi$ and $\psi$ ) of a protein sequence can be determined by potential energy and torque moment analysis. The $\beta$-hairpin, with two stands interlinked with folding characteristics of proteins, shows a two-state transition. Further analysis established that thermodynamic stability and fast folding kinetics are also interconnected. To avoid the kinetic traps, the co-translational folding and Cterminal residue interactions must be evaluated. The rare codons that affect protein assembling can be crucial for folding. But, how do the proteins constrain from non-native (fully unfolded) structures to native (folded) ones or distinguish as folded and out-of-register misfolded $\beta$-hairpin assemblies ( $N$, $\mathrm{N}$-terminus; C, C-terminus), and to perform for instance the side-chains of hydrophobic remain, are there above/below both hairpins, underlines as an unresolved fundamental question. ${ }^{29}$ The $\beta$ - strand has a predisposition to self-associate to form $\beta$-sheets, which are composed of several $\beta$-strands bound together by hydrogen bonds between the backbone atoms. Successive planes define angles $\phi$ and $\Psi$ of $\mathrm{O}, \mathrm{N}, \mathrm{C}$ and $\mathrm{H}$ atoms exist at a similar level, the conformation of a chain of $\eta$ amino acids replicated $2 \eta$ parameters, an energetically stable state. ${ }^{30}$ The high content of the hydrophobic 
residues in the amino acid chains play a crucial role to stop the misfolding. Therefore, it stabilizes inherent $\beta$-hairpin assembly. The resulting defective proteins may be unable to acquire requisite posttranslational modifications and assume their native conformations. There are many mechanisms (signal sequence cleavage, disulfide-bond formation/ isomerization, N-glycosylation, and glycosylphosphatidylinositol (GPI)-anchoring existed proteins. These transformations governed the various co- and post-translational alterations and modifications (Fig. 5).

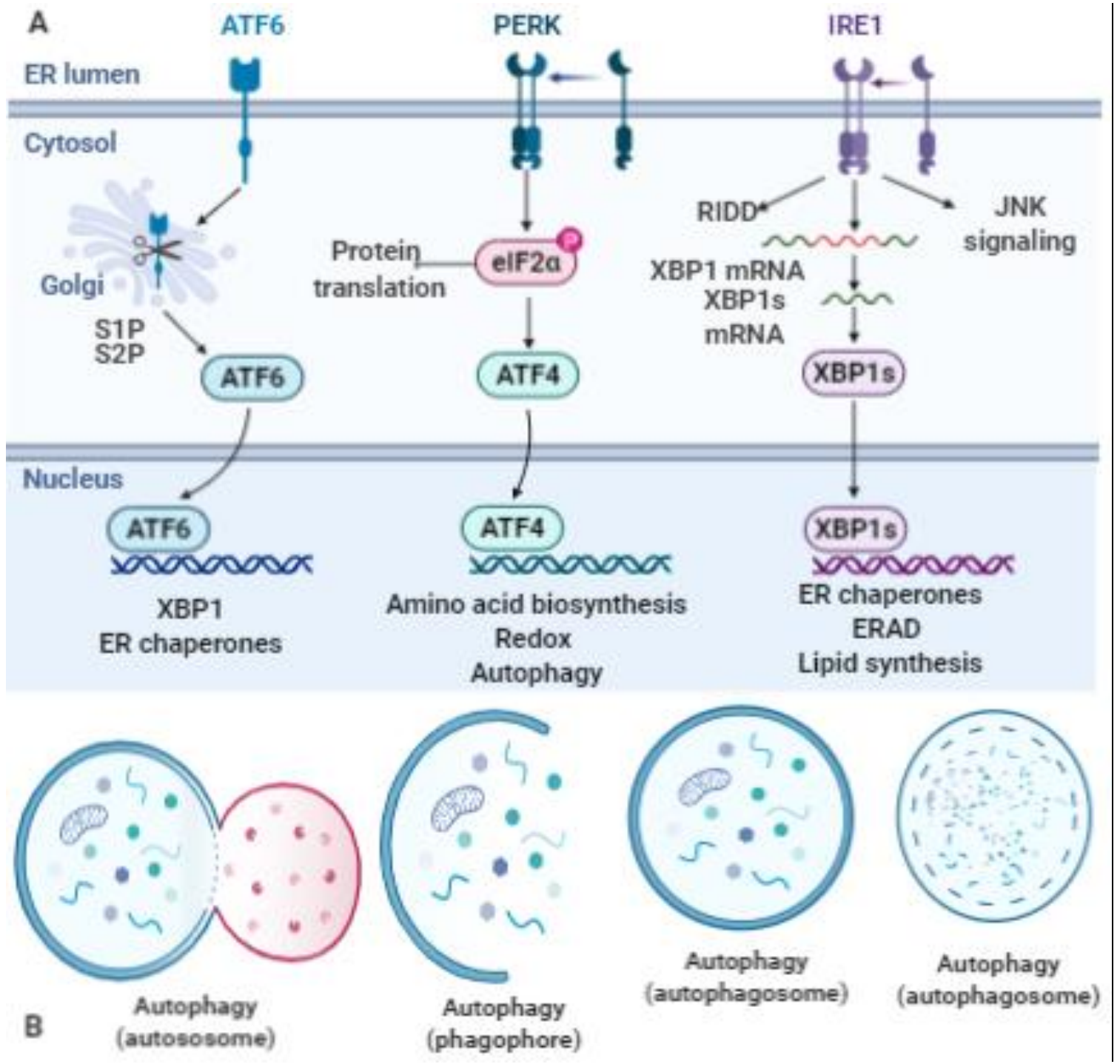

Fig. 5 Illustrations of the structure and molecular mechanism of stress signaling, unfolded protein response signal signaling. Defective proteins, requisite post-translational modifications. Co- and post-translational modifications, the lumen of the ER, and signal sequence cleavage, Golgi body, protein translation, autosome, phagophore, and autophagosomes. "Adapted and created with permission from [biorender.com]. Same is referred and acknowledged as per instructions". Thanks also for this template that is adapted and reproduced from Emma Madden Ph.D. Candidate, NUI Galway.

To elucidate the link between protein-misfolding/folding and chemical instability, there is a need to emphasize to expose the bond formation (oxidation) or bond cleavage (hydrolysis). ${ }^{31}$ Necessarily, for the interpretation of the denatured activity of cell with ongoing chemical conversion (reactions and mechanism, reaction intermediates, pathways of oxidation and reduction reactions, 
nucleophilic/electrophilic, molecular processes of chemical transformations, reactions discovery, pathways of enzymatic mechanisms, chemoinformatic processing of enzymatic transformations, reconstruction of metabolic pathways, computer-aided validation for proton and electron-driven reactions in protein-metallic catalysis, and computational analysis) must be strategically applied to elaborate chemical variability.

Generally, the oxidation of the proteins concerned with the various chemical transformations (metal ion catalyzed) or it will a photochemical. These are sourced from high energy $\gamma$-radiation or from organic additive. There are some many transformations (protein/peptide synthesis) initiated and governed by the metal catalysis. There are so many factors (consequent purification, bulk storing, and dosage formula) necessary for the successful metal-catalyzed oxidation. The amyloids, the final state of the aggregated proteins, represent the nature of it. The conformation of polypeptide distinguished by the dihedral angles $\phi$ and $\Psi$. The values of a single set of $\phi, \psi$ are sufficient to differentiate folded and misfolded types. ${ }^{32}$ Therefore, the dihedral angles are the key parameters to be underlined for improvement of proper understanding. The residue-shifted misleads folding in $\beta$-hairpin confirmation, which is varied by the configuration of the hydrogen bonds existed in the skeleton of a particular molecule. The underlined aspect of this interpretation is that the dihedral angles are not only the calculated features of the particular macromolecules, but also it defines the other characteristics of it. For example, these hold the equilibrium conformation that is a key factor to restructure the folded and misfolded states. The values of the $\varphi$ and $\psi$ angles can transformed into reaction coordinates. It is well evident that these parameters demonstrate the two-dimensional free energy landscapes. The question is, how do a $\beta$-hairpin misfold occurred, still persist unanswered. There are only three torsion angles that are as follows: $\varphi, \psi$, and $\omega$. Their mathematical values define the conformation of the skeleton. ${ }^{33}$ Further, these torsion angles interlinked with the angle of rotation around the formed bonds, $\mathrm{N}-\mathrm{C} \alpha$ bond. This angle notified as the phi $(\varphi)$ angle. There is one more rotation occurred around the bond defined as $\mathrm{C} \alpha-\mathrm{C}$. This angle marked as psi $(\psi)$. Furthermore, the rotation happened round the bond known as $\mathrm{C}-\mathrm{N}$ bond. This angle called as omega (w). There is a possibility that at one position or location any structure or residue of the secondary configuration has similarities with the values of the dihedral angle $(\varphi, \psi)$ existed within identified regions. These landscapes notified the existence of the secondary assemblies having very well defined values of the dihedral angles. These have two sub-types that are as follows (i) the right-handed $\alpha-$ helix, (ii) the left-handed $\alpha$-helix, and (iii) the $\beta$-strand. Therefore, the $\alpha$-helix is the main component of the protein states and solely responsible for the spiral-look. These arrangements further balanced 
by the parallel hydrogen bonds that formed between the skeleton atoms of successive residues of the protein.

\section{Pathological conditions: chaperones, ATP hydrolysis, and mitochondrial $\mathrm{Ca}^{2+}$ uptake}

Interference in proteostasis originates pathogenesis and initiates aging and the possibilities for the propagation of the degenerative diseases increased to start. To understand the mechanisms of chaperone and co-chaperones in both situations (regulating natural folding and the pathological conditions wherein misfolding of the proteins existed), is the key factor for the interpretation of sequences incidences. Generally, the chaperone assisted through the bind-and-release cycle because these are ATPases, and the necessary energy may be produced by ATP hydrolysis. ${ }^{34}$ The unrestricted heat forced chaperones to take part in protein folding at the expense of ATP. Molecular chaperones (Hsp60s, Hsp70s, Hsp90s, and sHsps) govern folding intermediates, aggregation, and persisted hurdles of the misfolding processes. ${ }^{35}$ Thus, these chaperones are directly responsible for the propagation of diseases and amyloid disorders. One of the varieties of these chaperones, Hsp70, existed in two states different states i.e. low/high-affinity ATP state and that also control folding catalysis. Therefore, ATP binding and hydrolysis are the key components for the proper functioning of co-chaperones, and chaperones connected and interlinked pathways, (Fig. 6). Thus, it's a very challenging task to pose the theoretical and computational methods for protein-surface examination. In this context, the authors identified applicability of different modeling and simulation techniques to address the issue. The quantum mechanics is about atom/molecules and utilized for the analysis of all-atom molecular mechanics by calculating free energy with a coarse-grained methodology. The $\mathrm{Ca}^{2+}$ activated metabolic enzymes by stimulating of energy-consuming routes in the cytosol during the processes of contraction and secretion simultaneously. In the meantime, the mitochondrial dehydrogenases stimulated concerned routes. These types of mechanisms were considered as the aerobic metabolism process that happened to complete the energy requirements of the active cell. Therefore, the route of the mitochondrial $\mathrm{Ca}^{2+}$ acceptance identified for proper understanding. ${ }^{36} \mathrm{The}^{2}$ pathological conditions governed by the mitochondria, which indicate the alteration in the $\mathrm{Ca}^{2+}$ signals and can be induced by the oxidative stress. These uncontrolled transformations change the performance of the organelle. It is evident that these unwanted activities can alter the structure of the organelle. As a final point, in some cases the cells have to enter upon apoptosis. The functioning of the mitochondria influenced by these degradations wherein some of the $\beta$-cells neighboring the 
secretory insulin granules act to stop proper functioning. ${ }^{37}$ These factors play a crucial role in the metabolism-secretion coupling.

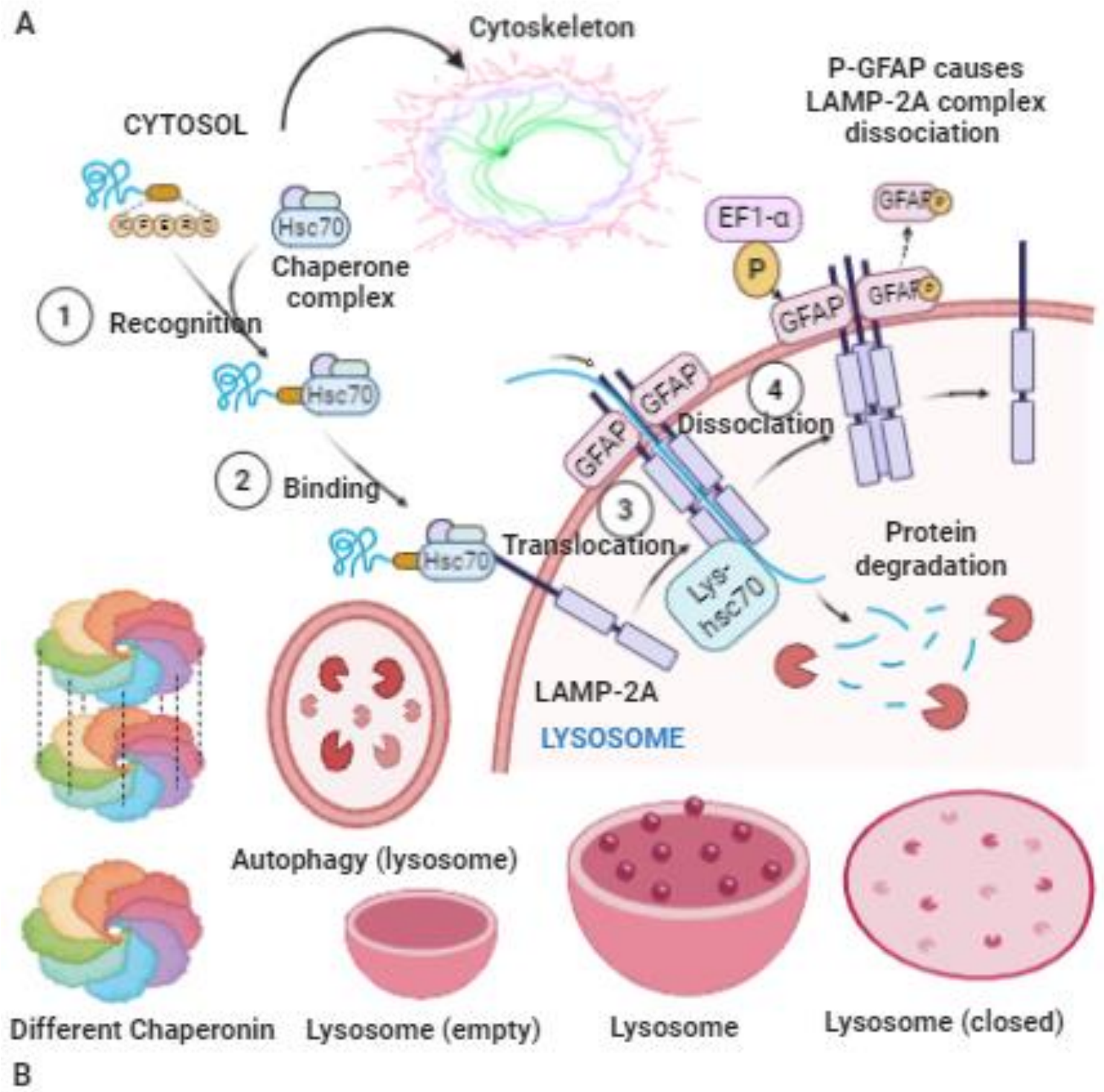

Fig. 6 Illustrations of the chaperone assisted, the bind-and-release cycle because, ATPases, and ATP hydrolysis. The heat forced chaperones, protein folding. These mechanisms to consume ATP. Molecular chaperones (Hsp60s, Hsp70s, Hsp90s, and sHsps) govern folding intermediates, aggregation and act as hurdles for misfolding processes. Propagation of diseases and amyloid disorders. "Adapted and created with permission from [biorender.com]. Same is referred and acknowledged as per instructions".

As in all the cell activities and transformation, the ATP supply the needed energy for the imitation of insulin granules and priming of exocytosis. The phenomena of the insulin exocytosis occurred when the ratio of the glycolysis in the $\beta$-cells amplified and as a result the percentage of the blood glucose reached as high. These transformations need a lot of energy that was supplied by the ATP and in the meantime, the ATP production increased. One more important transformation involved in it, and that is known as glucose-stimulation route. In this process, the $\beta$-cells secrete insulin that further initiates 
various signaling pathways. ${ }^{38,39}$ The $\mathrm{Ca}^{2+}$ signal originated from the mitochondrial activation play a role to end these chain events. Overall, these transformations and pathways governed the uptake of the glucose by the $\beta$-cells and the process of the insulin exocytosis.

\section{Mitochondrial $\mathrm{Ca}^{+2}$ homeostasis: decoding of extracellular stimuli}

The outputs of the mitochondrial biogenesis can be utilized for the pharmacological benefits. Therefore, mitochondrial $\mathrm{Ca}^{+2}$ homeostasis must be considered as a potential therapeutics to treat degenerative diseases existed in the mitochondrial for enhancing lifestyle. The analysis of the mitohormesis, and intracellular signaling, will develop a new methodology towards a new pharmacological target, and natural therapies. ${ }^{40}$ Mitochondria play a crucial role in governing a lot of pathways and chemical transformation. The signal transduction is one of the routes utilized by the mitochondria for the decoding. A verities of the intercellular actions such as an energy production route and apoptosis pathways ensued by extracellular stimuli. The modifications and alterations in the mitochondrial calcium $\mathrm{Ca}^{+2}$ homeostasis are the key mechanism through which a cell involved in various functions and processes. ${ }^{41}$ Any perturbation transpired in the mitochondrial $\mathrm{Ca}^{+2}$ homeostasis are responsible for the origin of pathological conditions. It is also depends on the verities of the cell. Mitochondrial impairment caused various neurodegenerative and neuroinflammatory disorders. ${ }^{42}$ To recognize these complications, the analysis and interpretation of the $\mathrm{Ca}^{2+}$ homeostasis mechanism definitely will a crucial factor to identify these complications. With the help of the molecular dynamics simulations, it is quite possible to investigate the biomolecular assembly and the concerned features of the dynamics going on at the atomic level. These methodologies are also capable to expose the complicated molecular processes occurred during protein conformations. Any other mechanism and pathways, for example the enzyme-reaction mechanisms and ligand binding ${ }^{43-}$ 45 routes happened during the protein folding and denaturation. Therefore, by the utilization of the computer and theoretical innovations, it is possible to shed some light on the routes and the pathways transpired during the protein folding. The same can be evident from the conducting the accompanied experiments employed in elucidating the folding machineries. These techniques may further utilize for the detection of other atomic detail. ${ }^{46}$ By employing a multidisciplinary approach, it may be possible to break the boundaries existed among various fields i.e. biochemistry, chemistry, medicine, and physics. These new themes displayed potential to prove their worth. The investigation of the landscape of the process of the folding energy pointed out intermediate constitutes and their nature. A proposed 3D model will notify the nature of the barriers and help out to overcome it. These 
investigations further help out to understand the mechanism of the amyloidogenic disease. These efforts can bring hope to find out the remedy for them. The outputs and procedures can be utilized to expose hidden routes and paths of hemodialysis-related amyloidosis. These pathological conditions are among the main causes which initiate aggregation in the sheet protein 2-microglobulin and the protein fibrils. ${ }^{47}$

Finally, there are various proteins such as $\mathrm{Bcl}-2$ family proteins, which control the process of mitochondrial $\mathrm{Ca}^{+2}$ homeostasis for proper regularization of the cells activates by the controlling of the permeability of the mitochondrial membrane. These specific proteins have various features and can intervene the various routes responsible for apoptosis. ${ }^{25}$ The decoding tactics of extracellular stimuli can further be useful. There are possibilities to develop such an anti-apoptotic interventions which are able to point out the concerned features of the $\mathrm{Bcl}-2$ protein family require for the possible development of the anti-ischemic therapy. These possibilities can be achieved by aiming to generate the anti-apoptotic functions or initiate loss in the pro-apoptotic function. If there were some therapeutic which will inhibit the permeability of the mitochondrial outer membrane, could be recommended to stop apoptosis. These strategies can be successful if a technique can control and alter the features of the $\mathrm{Bcl}-2$ family proteins. This can also be achieved by shielding the myocardium during any ischemia or reperfusion injury. ${ }^{48}$ These efforts may be fruitful for gaining the desired therapeutic for the clinical trials shortly. The main features of the formation of perfect folded $\beta$-hairpin assembly occurred in three steps (i) the turn formation, (ii) the hydrophobic core packing, and (iii) assembling of $\mathrm{H}$-bonds. ${ }^{49}$ The comparative analysis of the structural features of the misfolded/correctly folded protein reveals that there are three main differences. They have different turn configuration, ineffective filling of cross-strand hydrophobic side-chains, and altered hydrogen bonding existed in the backbone. These features are present between antiparallel strands that were initiated by the shifting of the concerned residue. It is quite complicated to determine the folding/misfolding pathway of the mechanisms of $\beta$-hairpin. ${ }^{50}$ It is quite challenging to analyze the misfolded assembly by comparing it with the native folded structure of $\beta$-hairpin with the folded one of the $\beta$-hairpin redesign experiments. From the data analysis of the topologies and measurement of hydrophobic residues of the amino acid of these two states, that can be underlined for such interpretations. As a low percentage of the hydrophobic residues in amino acid is the sign of the misfolding possibility. But if this percentage is high, then it improves the firmness of the native $\beta$ hairpin assembly. 


\section{Mitochondria-based pharmacological strategies: decoding of oxidative insults}

The processes of signal transduction are supported by mitochondria because it is capable of decoding numerous and different categories of signals of extracellular stimuli into action (intracellular actions, energy production, and cell death). If the process of mitochondrial calcium $\left(\mathrm{Ca}^{2+}\right)$ homeostasis originated, it will further initiate several other pathological conditions. Recently, it was identified that some proteins are involved in mitochondrial calcium homeostasis and now, this is the source of this discussion which will be helpful in the innovation of the mitochondria-based pharmacological strategies. ${ }^{51}$ Therefore, the failure of the mitochondrion activities initiates metabolic and nervous disorders. But the pathways of mitochondrial biogenesis, and dynamics, mitophagy have the potential to check such imbalances in the functioning of the mitochondrion. Any therapeutic strategy, by which the regulation of mitochondrion activities can be controlled, it will be a crucial innovation for any kind of drug discovery for the treatment of rare diseases. The mitochondrial oxidative phosphorylation and glycolytic processes influenced by the disorders in oxidative metabolism, further enhance neurodegenerative processes. Again, the initiated inequality between the inflammatory cytokines and cellular energy makes it worse. ${ }^{52}$ Thus, the urgencies for repairing and cleaning of the mitochondrial machinery of the cell must be well addressed to remove the perturbations in cellular proteostasis, proteolysis, and endocytosis. Despite, the computational predictions to protein conformational errors, accumulation of errors in displacement deteriorated cell environmental conditions, coding failure in the mitochondria-to-nucleus signaling pathway (trafficking defects, mitochondria-to-nucleus signaling pathway, neuronal dysfunction, endothelial dysfunction, macular degeneration, proteasome activity, and ubiquitination) and lots of others alike hidden pathways have never been experimentally enumerated, therefore, these mentioned mechanisms will be the foremost area to mark. ${ }^{53}$

This review article emphasizes on the specific and aforementioned aim, how to stop the cell from not doing denaturing activities and if persist how to stop them to clean the cells. There is an imperative necessity to tailor groundbreaking strategies for cell cleaning, with the foremost ambition to alienate the spin-off of cell necrosis, and apoptotic bodies from the cellular environment to recuperate as natural operability. In these circumstances, the organs lost their proper functioning because of these degradation activities which further initiates a lot of deteriorating disorders. Especially, these degenerations activities promote apoptosis in non-proliferating cells. The ATP synthesis occurred in the mitochondria interlinked with the generation of the reactive oxygen species. The superoxide $\left(\mathrm{O}^{2-}\right)$ formation also observed on the route of the respiratory chain. ${ }^{54}$ Subsequently, the superoxide initiates

unwanted oxidants (peroxynitrite, hydrogen peroxide) and fast responding hydroxyl radicals. The 
molecular mechanism of the route of the apoptosis identified and underlined the impact of the mitochondria in decoding processes. Finally, these transformations notified the oxidative insults. In the meantime, the proteins released catalyzed by the presence of cytochrome c which acted as cofactors of effector caspases.

\section{Ubiquitin proteasome pathway, DNA repair, and endocytosis}

The cell defense network consists of genome and proteome, any degradation in their concerned pathways or components further triggered various pathogenic states and aging. Thus, the blueprint of these pathways of genome and proteome damages must be underlined for further research. The analysis and interpretation of induced DNA damage must be done on an urgent basis. Because these phenomena played a key role and acted as a deciding factor in shaping the cellular landscape. These observations must be induced with the strategies planned for the innovation of the therapeutic modalities. Why the molecular pathways are forced cell aging, pathogenesis, and apoptosis? How does proteostasis interlink with translation and oxidative damage? And how do the pathways of the protein folding, molecular chaperones functioning, and protein quality control deal with the irreparably damaged proteins? All these undiscovered happenings interlinked with each other which to be investigated and exposed. Few of them performed for the betterment of the cellular functioning. ${ }^{55}$ One more important set of cellular processes is the controlling of the chaperons activities, how does it happen to save the healthy proteins, required, and in demand by the cells during the impactful action of the molecular chaperons. An imbalance between the cellular process of the generation of good proteins, and damaged proteins initiate apoptosis. It was reported that if there are more chaperones or proteasomes exist, then it will be good for a long age. The rate of the accumulating cellular damages and the cell's normal proteostasis has directly interrelated with aging. Therefore, there is an urgent need to innovate technologies for repairing the damaged $\mathrm{DNA}^{56,57}$ as, these DNA is repaired pathways is directly responsible for genome stability. ${ }^{58}$ The requirements of quality proteins are there to repair the DNA, and this is the main concern and that to be underlined as the necessities of cell cleaning. Interestingly, naturally, there are some proteins exist in the cellular environment, and has the potential to avoid misfolding, degradation, and aggregation and can be used to repair the DNA damage for proper regulation. It has been established that the molecular chaperones played a crucial role in repairing damaged DNA. During the pathological condition, DNA

repair machinery covered defects existed in protein quality control and vice versa. ${ }^{59}$ It is widely accepted that genome controlled cellular perturbations and do necessary maintenances. On the other 
hand, the reassembled proteome's repaired the genome as well as retained proper cellular functioning. The damages at the structural and genomic levels occurred due to the oxidative proteome. Genome mutations influenced the reactive oxygen species, oxidative DNA damage, ${ }^{60}$ and irretrievable oxidative proteome destruction. ${ }^{61}$ These pathways directly predisposed the efficacy and precision of the cellular processes and as a result, degeneracy arose. The unfolded protein response influenced the transcription of the gene and participates in DNA repair and cell-cycle regulation. Therefore, it is very helpful in the development of therapeutic modalities for the repair of the damaged DNA. ${ }^{62}$ Uncontrolled pathways initiate abnormal protein deposition, and the inability of the protein quality control, proteostasis, to deal with it may cause a lot of diseases. Therefore, the protein oxidation and damaged DNA directly responsible for the protein destruction. It may be assumed that DNA and DNA-binding proteins affected gene quality. To prevent, and control DNA damaging, ${ }^{63}$ all cells have kinase-based signaling networks ${ }^{64}$ as safeguards and it is a critical factor for apoptotic machinery, (Fig. 7). Genes participated in these cellular processes by transcriptional activation.

The ubiquitylation regulated a dynamic cellular process for stability and cellular homeostasis. The ubiquitin-signaling stressed the ubiquitin-conjugation machinery for proper surveillance. ${ }^{65}$ Monoubiquitination associated with numerous cellular functions (endocytic trafficking and DNA repair) and trafficking cargoes, it underlined the correctness of the endocytic route, receptor endocytosis, and degradation, and act as Ub-receptors in the endosome. By being passive and not depending on specific molecular components, these principles unveiled the general mechanisms (adaptation and rearrangement) of a significant portion of the cell membrane area. ${ }^{66}$ Furthermore, mutations also caused misfolding, and the nonfunctional forms of proteins are there to accumulate it. It proved that many pathological conditions are fundamentally rooted in protein-folding problems. All the cells must have to resolve them on a priority basis to maintain their functioning and integrity. The cellular proteome is a complex microcosm of structural and regulatory networks required for continuous surveillance and do modification to meet the dynamic needs of the cell. ${ }^{67}$ Genetic alterations, ranging from chromosome imbalance to oncogene initiation influenced the rate, fidelity, and ability to perform in protein biogenesis. 


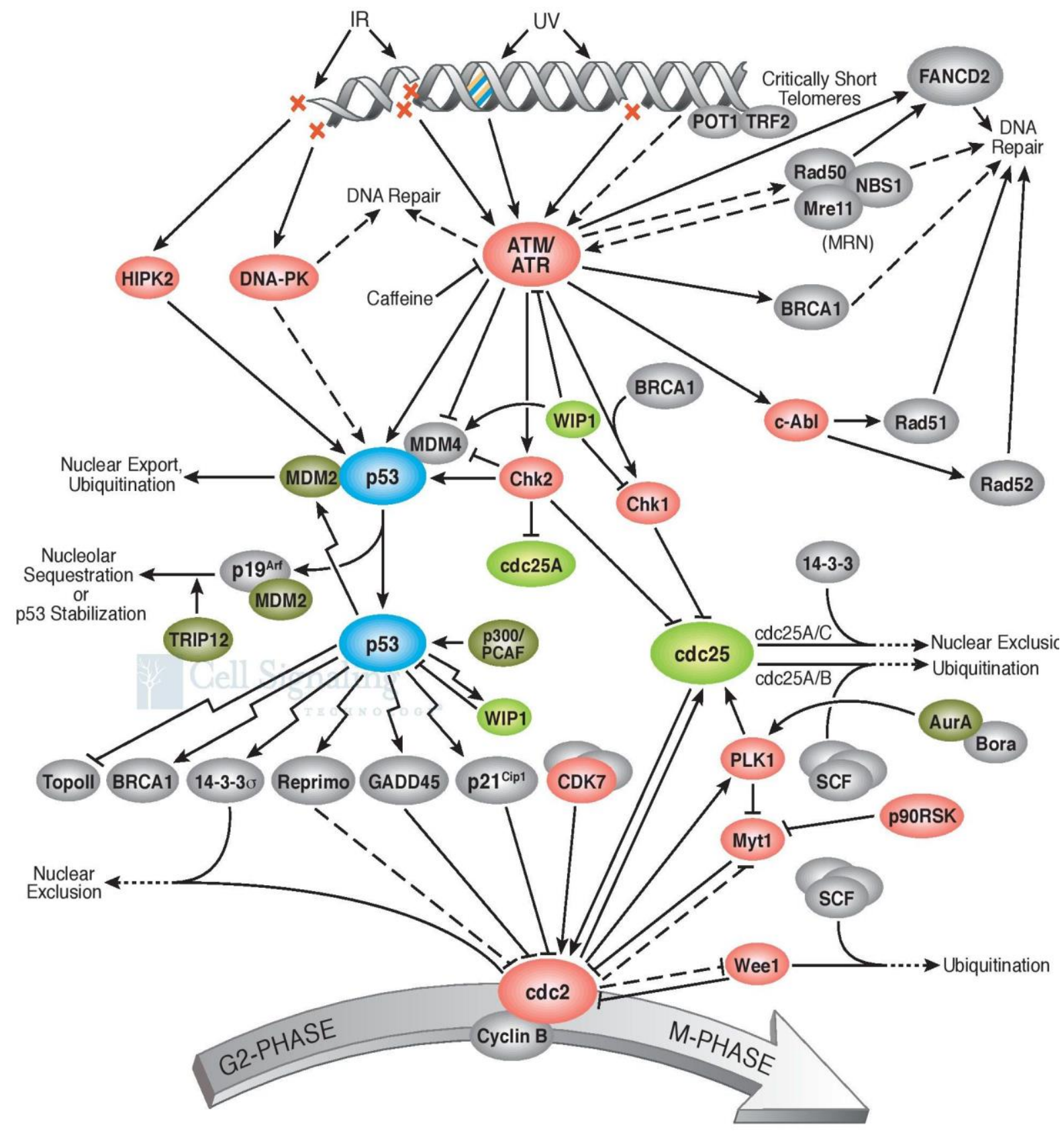

Fig. 7 Illustrations of the repairing of the damaged DNA. The DNA is repaired pathways and genome stability. The proteins needed to repair the DNA. Molecular chaperones and repairing DNA. "Adapted with permission from [www.cellsignal.com] and the same is acknowledged.

Any imbalance in the proteome functioning often initiates the degradation of cellular functioning and the environment. As, the ubiquitin pathways participated in the protein alteration and modification and regulate DNA and endocytosis ${ }^{68}$ than, any imbalance or build up the aggregation in the misfolded proteins initiates various kinds of neurodegenerative diseases. Therefore, the ubiquitinproteasome mechanism alter and destroy a lot of varieties of proteins participate in definite signals, or 'degrons'. 


\section{Serine-protease inhibitors, and ring-finger domain}

Folding patterns of proteins adopted more stable thermodynamic states. The serine protease inhibitors modified several biological mechanisms (blood clotting and inflammation) and highly capable of altering their configuration. The ability of conformational conversion allowed them to trap protease. To study the conformational patterns and adoption of the serpin, $\alpha 1$-antitrypsin will be further prolific with the concern of their ability to check the pathogenic states. ${ }^{69}$ During protein folding and their related enzymatic activities, the pro-peptide did not participate in it and was removed intramolecularly by autoprocessing. Thus, there is a need to address and rectify the possibilities, how an intramolecular chaperone defines the role of the pro-peptide in protein folding. ${ }^{70}$ As serine protease inhibitors are involved in conformation modifications desirable for biological function, but is there any other structural features (buried polar groups, cavities, and over-packing of side-chains) to oppose proper folding. Thus, the processes of the folding of serpin polypeptides can be erred and resulted in an inactive conformation. There are amino acid subunits be responsible for clear unfavorable interactions, stabilize substitutions of $\alpha 1$-antitrypsin, and also enhance thermostability. ${ }^{71}$ Serpins also defend immune cells from the attack of cytotoxic proteases. It is well known that the serpin mechanism is highly stable during disease-associated mutations. This highlights their importance in maintaining the balance and health of inhibitor conformation. Conformational stability and changes persisted due to the difference in higher free energy states and lower free energy states support during their performance in physiological functioning.

Sometimes, these conformational changes influenced free energy to inhibit target proteases by removing the protease. For example, during blood coagulation, some varieties of serpin regulate thrombin and other proteases, and any dysregulation, the misfolding leads towards thrombosis and other diseases. The serpins have $\mathrm{N}$ terminus and $\mathrm{C}$ terminus with disulfide bonds, and their bonding patterns govern their metastable states. ${ }^{72}$ For an effective and efficient folding, $\mathrm{N}$ and C-bonded glycans support the molecular chaperones. ${ }^{73}$ These analyses contribute to finding out the disease phenotypes. The proteases and their natural protein inhibitor ratio are a crucial factor in a balanced biological system. There are a lot of small folds in protein inhibitors having disulfide bonding for governing the oxidative folding. For the prediction or analysis of folding patterns of the two-domain protease inhibitors, anyone may expose their oxidative folding pathways. The intermediates of the protease inhibitors and their molecular mechanisms expose the oxidative folding of the disulfide-rich proteins. These interpretations will lead the researchers towards the $\mathrm{N}$-glycans functioning, 
physiological exchanges, and the biochemical transformation. ${ }^{74}$ The role of the $\mathrm{N}$-glycosylation mechanisms on folding, (Fig. 8) and the extracellular expression must be underlined.

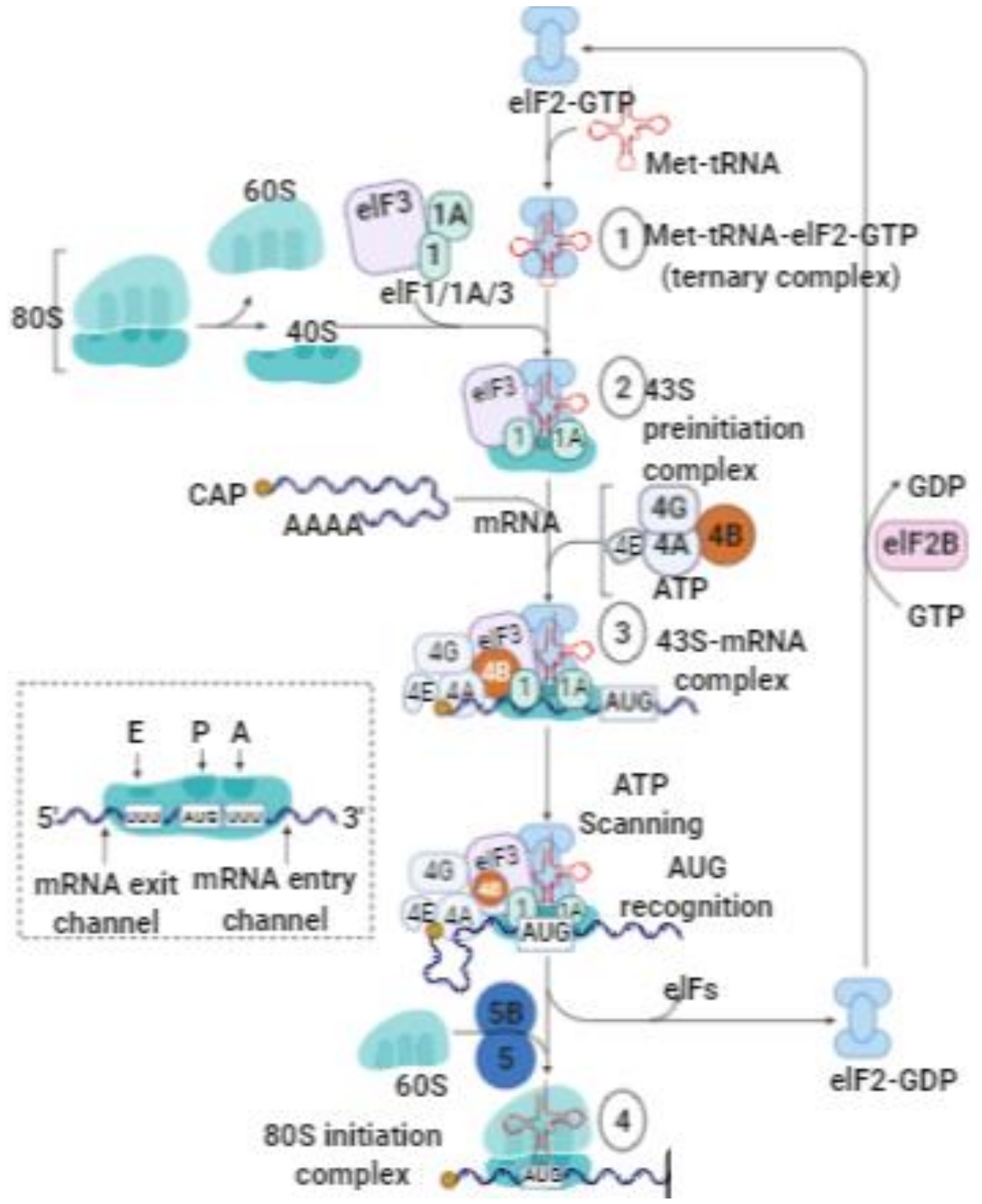

Fig. 8 Illustrations of the protein translation cascade, the $E R$, signal sequence cleavage and the link between protein-misfolding/folding and chemical instability, bond formation (oxidation) and bond cleavage (hydrolysis). "Adapted and created with permission from [biorender.com]. Same is referred and acknowledged as per instructions".

Thus, it inhibits Corin and enters a peptide cell surface expression and prothrombin secretion. ${ }^{75}$ The calnexin expression controlled Corin cell surface expression and prothrombin secretion in their protease domains. Functional modification of the RING finger and the ubiquitin system, (catalyzing cofactor and amino acid biosynthesis) having adenylating E1-like enzymes and ubiquitinlike proteins (Ubls) for the possessing of E2 enzymes. The class of RING finger E3s is operational with E1s, E2s, JAB domain peptides, and Ubls. These E1s, E2s, and RING fingers change little by little. They have highlighted sequencing with the creation of an oxyanion-hole in E2s and the C-terminal in the 
E1 enzyme existing with E2 in the active site. These RING fingers showed different functions and not all of them are participating in Ub-related functions. The analysis of kinetic accessibility during folding and refolding processes may be helpful to understand folding patterns. To understand the protein conformation conversions and interactions which are the central domain of the rational design of new tools in the field of interdisciplinary research (biomaterial sciences, nanotechnology ${ }^{76,77}$ and nanomedicine ${ }^{78,79}$ and findings in biochemistry, chemistry, medicine, and physics) ${ }^{80,81}$, the theoretical interpretations and analysis of the protein must be explored. Further, the role of the protein-surface binding mechanisms, ${ }^{43,45}$ for originating stimulation with their binding specificity at the different surfaces must be simultaneous with it. Obtained results acquiesced altered models with the detailed features of the thermodynamics and kinetics of adsorption. ${ }^{82}$ These models achieved by employing the computer programming to explore the possibilities and routes followed by the dynamics of the proteins and interweaves materials. These methods also interrupted the existed similarities and dissimilarities. Further, it underlined the role of the employed models and force-field tactics that were utilized for the analysis of the physical properties and the chemical/physical interactions at the material surfaces. These results can differentiate the concerned features of the biological molecules, while it were compared with other materials. ${ }^{83}$ In particular, the force fields and other models employed for the biomolecular simulations ${ }^{84}$ often not directly applicable to detect the feasibility of the surface simulations and vice versa.

\section{Undiscovered pathways and mechanisms: the what, the why's, and the how's}

The outlines of the ER-associated degradation pathway and the mechanism of tracking pathways are highlighted for further analysis. The mechanism of tagging of substrates ubiquitin by the coordinated action of cell organelles/proteins/enzyme/ATP/gene alteration/DNA/PQCPs/cell cleaning are among the key pathways to be underlined. The analysis of the presence of intra- and extracellular deposits of proteins which altered conformation and posttranslational modifications by prior attachment to polymers of ubiquitin, must be specifically done and applied to the interpretation of various pathways. The analyses of the pathologies of the protein misfolding underlined the intra/extra cellular aggregation that was executed at the time of the origin of the diseases in the proteins. One more requirement of these studies and interpretation is to find out the terminuses existed in the secretory pathway. These examines also discovered the impact of the non-native and unassembled subunits existed in the multimeric proteins degraded through ubiquitination. These phenomena happened to the residues that were marked as the proteasomal degradation signal. These are the hidden areas 
that must be identified and underlined in the near future. The detection of diseases associated with ERAD substrates is featuring the importance of the analysis of these pathways and mechanisms with an emphasis on the factors that catalyze the distinct activities. Therefore, how the illustration employed to explore the cell cleaning necessity and interrelated cell processes under proteotoxic stress and situations, must be investigated for a suitable answer. These portraits of the different routes lead and explore the understanding of the tempering mechanism transpired in the translation rate. The sequestration of mRNAs encoded cleaning routes adopted in the proteins. Here, the stress granules assist redirection of the energies in the direction of crucial pro-survival pathways $?^{85}$ Molecular chaperones and associated factors recognized and targeted the substrates for retrotranslocation to the cytoplasm, wherein they are degraded by the ubiquitin-proteasome machinery. How does the molecular machinery identity the main proteins are involved in the handling of mitochondrial $\mathrm{Ca}^{2+}$ traffic, must be analyzed and findings must be utilized for opening new fascinating and ambitious avenues for the innovation of mitochondria-based pharmacological strategies. The Ca-activated, and ATP-independent proteolytic process tangled in the cysteine proteases term as calpains ${ }^{86}$ How do these proteases activate during cell injuries and their role in rises of the cytosolic $\mathrm{Ca}^{2+}$ rises?, It must be investigated. What to do to distinguish between the folded and out-of-register misfolded $\beta$-hairpin structures ( $N, N$-terminus; $C, C$-terminus), and the side-chains of hydrophobic residues above/below the both hairpins?, The answer of these queries will be a key option to redefine several features of it. Why are the $\beta$ - strand has a predisposition to self-associate to form the $\beta$-sheets, which consists of several $\beta$-strands bound together by hydrogen bonds between the backbone atoms $?^{87}$ there is a need to search the suitable cause responsible for them. For instant, this can be defined by a single set of $(\phi, \Psi)$ values, for both folded (F) and misfolded (M) states. Therefore, the dihedral angles of the turn residues might be used to differentiate these two distinct states.

Thereafter, in the configurations of the misfolded $\beta$-hairpin, the residue-shifted happened to regulate it. These shifts in the residue varied during the assembling of the hydrogen bonds in the pattern. One of the configurations, the omega $(\omega)$ existed around C-N bond formed secondary types. All the successive deposits persisted in these engagements displayed similar dihedral angle $(\varphi, \psi)$ values in the identified regions. ${ }^{88}$ The main component of protein assembly is $\alpha$-helix and identified easily by the spiral-look. It is important to know that how structural features stabilize the process of the formation of $\mathrm{H}$-bonds. These hydrogen bonds situated between the atoms of successive residues of the proteins. These descriptions analyzed the origin of the misfolding transpired in $\beta$-hairpin. It further 
supported by noticeable investigations in $\beta$-hairpin folding ${ }^{29}$ It is a challenging task to detect the impact of the misfolded proteins in the creation of the $\beta$-hairpin hairpin (naturally bent arrangement) during the redesigning of the experiments. There are different types of co- and post-translational modifications happened in the lumen of the ER.

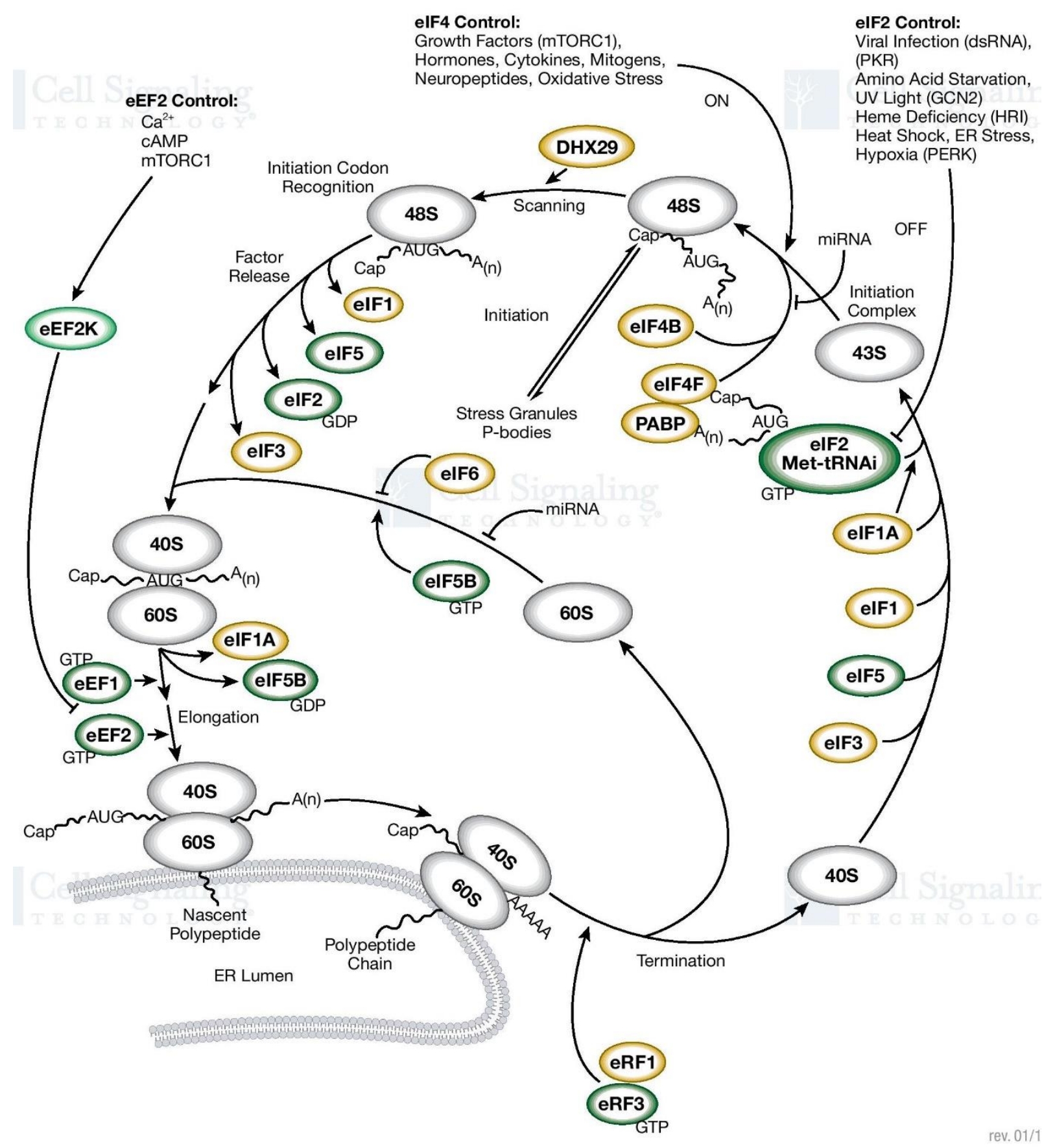

Fig. 9 Illustrations of the translation control over various co- and post-translational modifications in the lumen of the ER (signal sequence cleavage, N-glycosylation, glycosylphosphatidylinositol (GPI) -anchoring, and disulfidebond formation/isomerization). "Adapted with permission from [www.cellsignal.com] and the same is acknowledged.

The signal sequence cleavage, N-glycosylation, glycosylphosphatidylinositol (GPI) -anchoring, and disulfide-bond formation/isomerization) are the main transformations routes and pathways 
necessarily looked-for to further scrutinize. To elucidate the link between protein-misfolding/folding and chemical instability, there is a need to explore the mechanism of the bond formation (oxidation) or bond cleavage (hydrolysis), that is most usually applied (Fig. 9). These mechanisms originated naturally to play an important role in tissue injury, necrosis, and autolysis and such facts must be scrutinized. For further research. There are so many other unsolved mysteries persisting in these pathways which to be answered. For example, how does the greater percentage of $\mathrm{Ca}^{2+}$ in the lumen stimulate extracellular existence and responsible for the stable conformation implementation. These routes governed the secretive pathways of $\mathrm{Ca}^{2+}$ which initiated the activities of the calcium/calmodulin-dependent protein kinase II. These transformations transpired through biochemical changes and triggered apoptosis. The mitochondrial pathways and death receptors played a crucial role in it. ${ }^{89}$ The ubiquitin renewed the routes and the concerned pathways to modify and alter the proteins necessarily. It may influence the alternative (non-degradative) roles of ubiquitin in the proper regulation of the routes and pathways of DNA repair and endocytosis. There is a necessity to expose these processes, and know that how do these mechanisms play crucial role in the regulation of events as recognition, unfolds, and cleaving of proteins. The process of mitochondrial $\mathrm{Ca}^{2+}$ homeostasis identified to apply the therapeutic tactics to treat several disorders. These approaches proposed new themes to find out suitable options biochemically. To identify the molecular process involved mitochondrial $\mathrm{Ca}^{2+}$ traffic and its further proper utilization for opening new channels in the innovation and development of the mitochondria-based pharmacological strategies are the main goal of this review.

How to distinguish between those proteins that have definite degradation signals, or degrons?, how to understand E3 ligase at the $\mathrm{N}$-end rule pathway that has inner lysine residue?, how to point out the unstructured $\mathrm{N}$-terminal extension which are accountable for ubiquitination?, how to find out those substrates of ubiquitination providing a degradation signal (degron)?, what are the main components of these signaling transformations?, and how to identify an N-terminal type 1 disrupting residue or type 2 destabilizing residues?. These are the main queries to be talked and answered urgently. ${ }^{90}$ More, similar questions like why do the alteration of the $\mathrm{Ca}^{2+}$ signals influence the functioning of the mitochondria in various pathological conditions? and how does oxidative stress generate consequences responsible for initiating apoptosis? These hidden mechanisms and their related facts must be revealed. Such inquiries help to focus on the urgency of development of the advanced mitochondrial based therapies as a natural remedy. The Ubiquitin proteasome pathway involved in anonymous, unidentified, and hidden pathways of unconventional (non-degradative) 
functions for the regulation of apoptosis. The research findings on enzymatic components link chains of $\mathrm{Ub}$ onto proteins include the E1, E2, and the third enzyme, E3 which is a key factor in terms of the specificity of proteolysis at the expense of energy in the form of ATP. These interpretations will crucial to explain the proper pathway of ubiquitin-activating. ${ }^{91}$ Neurodegenerative diseases influenced badly heme biosynthesis, oxygen sensing, mediate calcium homeostasis, and cell growth. Factors such as responses to stress, extracellular modulators, immune disorders, and inflammation must be considered as major risk causes during higher incidence and initiate aging. The enzymes/metal catalysis and cellular environmental stress are among the critical reasons for the destruction of cell constituents during apoptosis. To underline the needs of this discussion, in this review article, the authors recommend the following key points that to be addressed (1) the boosting phenomenon of the cellular defense structures in the topology of the misfolded proteins, (2) identifying strategies for survive failures of protein quality controls, (3) causes of the proteasome failure, (4) find out the persisting miscarriage happened during quality control in mitochondrial, ER, nuclear and ribosomal PQCPs, (5) identify the enzymes which can be catalyzed by the chemical mechanism, it will open doors to understanding how do the pathological protein quality control catastrophes reduce protein synthesis to increase folding. To detect such pathways that were delinked with the protein synthesis, there is an urgent need to identify such veiled, anonymous, and unidentified causes, which are solely responsible for increasing folding.

\section{Role of optical tweezers in protein folding}

The key causes of any error transpired in the regular and natural phenomenon of protein folding underlined for a proper analysis. It is very important to know, how does it happen and what are the mechanisms behind it. By utilizing optical tweezers, it is easy to find out the main causes responsible for these errored folding along with their concerned features. ${ }^{92}$ Therefore, the involved pathways and related parameters can be investigated and dignified by interrupting the extract folding energies and the single-molecule measurements. Further, the optical tweezers are quite helpful in determining the topographical changes, linear to three dimensional, originated by the errored sequencing in a dynamic equilibrium. This high-resolution technique based on single-molecule manipulation exposes the protein folding of a single protein or protein complex located between two beads. Their existence was identified by the optical traps and pulled as a transition. The analysis of the changes and erred persist in protein topologies during conformations and energies of folding intermediates along with associated transition kinetics will be the key. The optical tweezers played a crucial role to unearth the 
interlinked pathways necessary for the investigation of the folding of proteins and protein complexes, including data analysis, and folding energies of the single-molecule measurements. Generally, the optical tweezers detect the time-resolved extension of a single macromolecule which is a forcedependent property within two trapped beads. ${ }^{93}$ Thus, it deals with the measurements of various characteristics related to the folding intermediates, energies, and kinetics of the concerned macromolecule. Earlier, the reaction coordinates applied to identify the witnessed folding transitions that superficially define the folding without considering the impact of the force. But, in this methodology, the total extensions, energies, and transition rates of the proteins are considered for the calculation as a function of force to find out the best-fit conformations in related folding states. Folding of macro proteins happened in the translation process to discontinue inter-domain misfolding. The aspect of this analysis is to observe the folding intermediates and portrait sequential folding pathway originated from the C-terminus. Simply, the forces govern the structure and dynamic of these biomacromolecules by enabling folding, stirring the assembly, and determining the conformational fluctuations. Optical tweezers analyzed the molecular motors translocate DNA features that are ATP-dependent chromatin remodeling to re-structure the chromatin. These findings evidenced an impactful role and the allied features of high-resolution optical tweezers during the quantitatively illustrating of the multifaceted and multi-scale dynamics of biomacromolecules.

\section{Conclusion}

A research path applied to find out the appreciate errors in systems, error correction mechanism, and early etiological foundations of health and diseases like protein conformational disorders (PCDs), trafficking defects, mitochondria-to-nucleus signaling pathway, cardiovascular diseases, neuronal dysfunction, endothelial dysfunction, diabetes mellitus, aqueous-deficient dry eyes (ADDE), agerelated macular degeneration, cataract, proteasome activity, neurodegenerative disease, pick's disease, and ubiquitination in the pathogenesis of human diseases. The analysis and the interpretation of (1) Mechanism of organelle-specific PQCPs, (2) Cellular strategies to scrutinize proteins at the endoplasmic reticulum (ER)-Golgi interface, (3) Ribosomal perspective on proteostasis at the expense of ATP hydrolysis, (4) Molecular chaperones-therapeutic approaches, (5) Calcium-activated potassium channels, (6) Degradation signal (degron)- Variations in degradation signals, (7) Pathway of the stress to cellular homeostasis, (8) Building blocks and molecular dissection of the secretory pathway, and (9) Genetic alterations, proteostasis networks are very prolific that were underlined and highlighted in the objective of this review article. Further, authors tried to cover important pathways and different 
mechanisms of (1) Metal-protein interface originated from the interaction that is similar to the Lewis acids/base reaction and the other similar regulators happened during the interaction of metal-, ligand-, and enzyme-metal complexeation, here all the induced metals act as an electron donor or acceptor as per the need of the interface/reaction/interaction (2) Principal types of machinery maintaining proteostasis-ubiquitin proteasomal and lysosomal autophagy systems (3) Cell survival responses to environmental, stresses, oxidants, metabolism, and signal transduction (4) How cells adapt proteasome assembly under stress conditions, (5) Boosting cellular resilience to protein misfolding, (6) Identifying strategies for proteasome activation by natural or chemical compounds, (7) Endothelial apoptosis, angiogenesis and proteasomal degradation, (8) serine-protease inhibitors, and (9) Ring-finger domain. Important suggestions were incorporated to identify such hidden pathways and mechanisms occurred within the cellular compartment wherein the protein misfolding and the error in the mitochondria-to-nucleus signaling pathway merely responsible for the degradation of whole types of machinery and deteriorated cell environmental conditions. Further, it was suggested that the findings of the organic reaction mechanism, molecular alterations, metallic/enzymatic catalyzed bio-inorganic/organic transformation, computational spectroscopy and predictions, and interdisciplinary expertise's experience are worthwhile utilized for these interpretations. Finally, it was believed that these efforts will be very useful to delineate the hidden mechanisms.

\section{Acknowledgments}

One of the authors (Rajiv Kumar) gratefully acknowledges his younger brother Bitto for motivation. The authors acknowledge bio render for providing facility to illustrate the diagrams. The authors acknowledge the support towards the reproduction of the illustrations (figure 2, 4, 7 to 9) as a courtesy from Cell Signaling Technology, Inc. (www.cellsignal.com).

\section{Author contributions}

RK supervised and wrote this review article. $B C, K G$, and $M C$ suggested revising the manuscript. All authors read and approved the manuscript.

\footnotetext{
Author details

${ }^{*}$ NIET, National Institute of Medical Science, India.

${ }^{2}$ Department of Chemistry, Aditi Mahavidyalaya, University of Delhi. Delhi, 110027, India.

${ }^{3}$ Materials and Manufacturing, School of Engineering, University of Wolverhampton, England, UK TF29NN. ${ }^{4}$ Institute of Nuclear Medicine and Allied Sciences (INMAS) Brig. S. K. Mazumdar Marg, Delhi, 110054
}

\section{Funding}


The authors thank the national institute of medical science for its financial support.

\section{Availability of data and materials}

Wherever necessary, relevant citations are included in the reference section.

\section{Competing interests}

The authors have declared that no competing interest exists

\section{References}

1 J. Gandhi, A. C. Antonelli, A. Afridi, S. Vatsia, G. Joshi, V. Romanov, I. V. J. Murray and S. A. Khan, Rev. Neurosci., 2019, 30, 339-358.

2 N. Hosokawa, I. Wada, K. Hasegawa, T. Yorihuzi, L. O. Tremblay, A. Herscovics and K. Nagata, EMBO Rep., 2001, 2, 415-422.

3 K. R. Thomas, K. J. Bangen, A. J. Weigand, E. C. Edmonds, C. G. Wong, S. Cooper, L. DelanoWood and M. W. Bondi, Neurology, 2020, 94, e397-e406.

4 A. H. Hsieh and J. D. Twomey, J. Biomech., 2010, 43, 137-145.

5 L. Grozinger, M. Amos, T. E. Gorochowski, P. Carbonell, D. A. Oyarzún, R. Stoof, H. Fellermann, P. Zuliani, H. Tas and A. Goñi-Moreno, Nat. Commun., , DOI:10.1038/s41467-019-13232-z.

6 S. I. Nishikawa, J. L. Brodsky and K. Nakatsukasa, J. Biochem., 2005, 137, 551-555.

7 J. Lavie, H. De Belvalet, S. Sonon, A. M. Ion, E. Dumon, S. Melser, D. Lacombe, J. W. Dupuy, C. Lalou and G. Bénard, Cell Rep., 2018, 23, 2852-2863.

8 M. P. Mayer and L. M. Gierasch, J. Biol. Chem., 2019, 294, 2085-2097.

9 S. Acharyya, Chem. Biol. Lett., 2020, 7, 99-112.

10 I. Moreno-Gonzalez and C. Soto, Semin. Cell Dev. Biol., 2011, 22, 482-487.

11 M. P. Jackson and E. W. Hewitt, Essays Biochem., 2016, 60, 173-180.

12 R. Dabur, B. Sharma and A. Mittal, Chem. Biol. Lett., 2018, 5, 63-99.

13 D. P. de Lima, T. N. Reddy, A. Beatriz, R. da S. Lopes, M. R. Marques, E. dos A. dos Santos, C. I. Nantes, M. de F. C. Matos, R. T. Perdomo, I. C. Pereira and S. S. Weber, Chem. Biol. Lett., 2018, 5, 41-54. 
P. Jadiya and D. Tomar, Genes (Basel)., 2020, 11.

S. Savickas, P. Kastl and U. auf dem Keller, Biochim. Biophys. Acta - Proteins Proteomics, 2020, 1868.

17 S. Pemberton, K. Madiona, L. Pieri, M. Kabani, L. Bousset and R. Melki, J. Biol. Chem., 2011, 286, 34690-34699.

Y. Feng, D. He, Z. Yao and D. J. Klionsky, Cell Res., 2014, 24, 24-41.

A. Kuma, M. Komatsu and N. Mizushima, Autophagy, 2017, 13, 1619-1628.

M. Ponpuak, M. A. Mandell, T. Kimura, S. Chauhan, C. Cleyrat and V. Deretic, Curr. Opin. Cell Biol., 2015, 35, 106-116.

L. L. Yan and H. S. Zaher, EMBO J., , DOI:10.15252/embj.2019101633.

P. Bragoszewski, M. Turek and A. Chacinska, Open Biol., 2017, 7.

F. H. Ma, C. Li, Y. Liu and L. Shi, Adv. Mater., 2020, 32.

R. S. Balaban, S. Nemoto and T. Finkel, Cell, 2005, 120, 483-495.

K. S. Lee and B. Lu, Front. Cell. Neurosci., 2014, 8.

L. Fernandez-Mosquera, K. F. Yambire, R. Couto, L. Pereyra, K. Pabis, A. H. Ponsford, C. V. Diogo, M. Stagi, I. Milosevic and N. Raimundo, Autophagy, 2019, 15, 1572-1591.

O. Moltedo, P. Remondelli and G. Amodio, Front. Cell Dev. Biol., 2019, 7.

S. Long and P. Tian, Sci. Rep., , DOI:10.1038/srep34481.

Q. Shao, J. Phys. Chem. B, 2015, 119, 3893-3900.

A. Shelar, P. Kumar and M. Bansal, in Biomolecular Forms and Functions: A Celebration of 50 Years of the Ramachandran Map, 2012, pp. 116-127.

G. VON HEIJNE, Eur. J. Biochem., 1983, 133, 17-21.

32

C. J. Bosques, S. M. Tschampel, R. J. Woods and B. Imperiali, J. Am. Chem. Soc., 2004, 126, 8421-8425. 
L. E. Kay, M. Ikura, R. Tschudin and A. Bax, J. Magn. Reson., 1990, 89, 496-514.

34 B. Panaretou, C. Prodromou, S. M. Roe, R. O'Brien, J. E. Ladbury, P. W. Piper and L. H. Pearl, EMBO J., 1998, 17, 4829-4836.

H. Sghaier, T. L. H. Ai, T. Horiike and T. Shinozawa, In Silico Biol., 2004, 4, 311-322.

Y. Feng, W. Li, J. Li, J. Wang, J. Ge, D. Xu, Y. Liu, K. Wu, Q. Zeng, J. W. Wu, C. Tian, B. Zhou and M. Yang, Nature, 2012, 491, 478-482.

D. Akhmedov, M. Braun, C. Mataki, K. Park, T. Pozzan, K. Schoonjans, P. Rorsman, C. B. Wollheim and A. Wiederkehr, FASEB J., 2010, 24, 4613-4626.

H. J. Hwang, Y. R. Yang, H. Y. Kim, Y. Choi, K. S. Park, H. Lee, J. S. Ma, M. Yamamoto, J. Kim, Y. C. Chae, J. H. Choi, L. Cocco, P. O. Berggren, H. J. Jang and P. G. Suh, FASEB J., 2019, 33, 10668-10679.

S. Sharma and V. Bhatia, Chem. Biol. Lett., 2020, 7, 215-224.

40

Y. Pan, Exp. Gerontol., 2011, 46, 847-852.

41

C. Giorgi, C. Agnoletto, A. Bononi, M. Bonora, E. de Marchi, S. Marchi, S. Missiroli, S. Patergnani, F. Poletti, A. Rimessi, J. M. Suski, M. R. Wieckowski and P. Pinton, Mitochondrion, $2012,12,77-85$.

S. Voet, S. Srinivasan, M. Lamkanfi and G. Loo, EMBO Mol. Med., 2019, 11, e10248.

V. Chandel, M. Srivastava, A. Srivastava, S. Asthana and D. Kumar, Chem. Biol. Lett., 2020, 7, 47-54.

D. K. Behera, P. M. Behera, L. Acharya and A. Dixit, Chem. Biol. Lett., 2017, 4, 1-8.

D. Sharma, M. Pathak, R. Sharma, N. Sharma, P. Tyagi, R. Chawla, M. Basu and H. Ojha, Chem. Biol. Lett., 2017, 4, 27-32.

M. Compiani and E. Capriotti, Biochemistry, 2013, 52, 8601-8624.

47 K. V. Chanu, D. Thakuria, R. Sood, A. K. Pateriya, S. Bhatia and S. Kumar, Chem. Biol. Lett., 2017, 4, 20-26.

R. G. Pillai, Chem. Biol. Lett., 2020, 7, 41-46. 
X. Yuan, M. D. Johnson, J. Zhang, A. W. Lo, M. A. Schembri, L. C. Wijeyewickrema, R. N. Pike, G. H. M. Huysmans, I. R. Henderson and D. L. Leyton, Nat. Commun., , DOI:10.1038/s41467-01803593-2.

50 F. Simona, G. Tiana, R. A. Broglia and G. Colombo, J. Mol. Graph. Model., 2004, 23, 263-273.

51 A. J. Kowaltowski, S. L. Menezes-Filho, E. A. Assali, I. G. Gonçalves, J. V. Cabral-Costa, P. Abreu, N. Miller, P. Nolasco, F. R. M. Laurindo, A. Bruni-Cardoso and O. S. Shirihai, FASEB J., 2019, 33, 13176-13188.

52 W. Y. Wang, M. S. Tan, J. T. Yu and L. Tan, Ann. Transl. Med., 2015, 3.

53 Y. Nakazawa, Y. Hara, Y. Oka, O. Komine, D. van den Heuvel, C. Guo, Y. Daigaku, M. Isono, Y. He, M. Shimada, K. Kato, N. Jia, S. Hashimoto, Y. Kotani, Y. Miyoshi, M. Tanaka, A. Sobue, N. Mitsutake, T. Suganami, A. Masuda, K. Ohno, S. Nakada, T. Mashimo, K. Yamanaka, M. S. Luijsterburg and T. Ogi, Cell, 2020, 180, 1228-1244.e24.

54 M. P. Murphy, Biochem. J., 2009, 417, 1-13.

55 Y. Tu, C. Chen, J. Pan, J. Xu, Z. G. Zhou and C. Y. Wang, Int. J. Clin. Exp. Pathol., 2012, 5, 726738.

56 S. K. Misra, P. Moitra, B. S. Chhikara, P. Kondaiah and S. Bhattacharya, J. Mater. Chem., 2012, 22, 7985-7998.

57 B. S. Chhikara, S. K. Misra and S. Bhattacharya, Nanotechnology, 2012, 23, 065101.

58 R. Oshidari, K. Mekhail and A. Seeber, Trends Cell Biol., 2020, 30, 144-156.

59 M. O. Turgeon, N. J. S. Perry and G. Poulogiannis, Front. Oncol., 2018, 8.

60 P. J. Bindu, T. R. R. Naik, K. M. Mahadevan and G. Krishnamurthy, Chem. Biol. Lett., 2019, 6, 813.

61 P. Sengupta, S. Dutta, A. Alahmar and U. J. A. D'souza, Chem. Biol. Lett., 2020, 7, 75-84.

62 D. Branzei and M. Foiani, Nat. Rev. Mol. Cell Biol., 2008, 9, 297-308.

63 S. YAlçın, P. Mutlu, G. Unsoy, R. Khodadust and U. Gunduz, J. Mater. Nanosci., 2020, 7, $29-35$.

64 B. S. Chhikara, S. Ashraf, S. Mozaffari, N. St Jeans, D. Mandal, R. K. Tiwari, Z. Ul-Haq and K. Parang, Molecules, 2020, 25, 2135. 
M. Hedglin, M. Aitha, A. Pedley and S. J. Benkovic, J. Biol. Chem., 2019, 294, 5157-5168.

S. Devi, J. Singh, V. Kumar and V. Malik, Chem. Biol. Lett., 2019, 6, 39-45.

P. Greulich and B. D. Simons, Proc. Natl. Acad. Sci. U. S. A., 2016, 113, 7509-7514.

68

Z. Cao, D. Goyal, S. E. Meiler, Y. Zhou and W. S. Dynan, Artif. Cells, Nanomedicine Biotechnol., 2019, 47, 2196-2204.

69

B. Gooptu, J. A. Dickens and D. A. Lomas, Trends Mol. Med., 2014, 20, 116-127.

70

T. Sawada, M. Yamagami, S. Akinaga, T. Miyaji and M. Fujita, Chem. - An Asian J., 2017, 12, $1715-1718$.

71

W. Zhu, L. Li, M. Deng, B. Wang, M. Li, G. Ding, Z. Yang, D. Medynski, X. Lin, Y. Ouyang, J. Lin, L. Li and X. Lin, FEBS Open Bio, 2018, 8, 1711-1721.

B. Wu, A. J. McDonald, K. Markham, C. B. Rich, K. P. McHugh, J. Tatzelt, D. W. Colby, G. L. Millhauser and D. A. Harris, Elife, , DOI:10.7554/eLife.23473.

J. A. Carver, A. B. Grosas, H. Ecroyd and R. A. Quinlan, Cell Stress Chaperones, 2017, 22, 627638.

L. Meuris, F. Santens, G. Elson, N. Festjens, M. Boone, A. Dos Santos, S. Devos, F. Rousseau, E. Plets, E. Houthuys, P. Malinge, G. Magistrelli, L. Cons, L. Chatel, B. Devreese and N. Callewaert, Nat. Biotechnol., 2014, 32, 485-489.

F. Schwarz and M. Aebi, Curr. Opin. Struct. Biol., 2011, 21, 576-582.

R. Kumar and M. Sharma, J. Mater. Nanosci., 2018, 5, 35-58.

R. Kumar, B. S. Chhikara, K. Gulia and M. Chhilar, Theranostics, , DOI:10.7150/thno.49619.

B. S. Chhikara, J. Mater. Nanosci., 2017, 4, 19-24.

79

B. S. Chhikara, R. Kumar, B. Rathi, S. Krishnamoorthy and A. Kumar, J. Mater. Nanosci., 2016, 3, $50-56$.

80

M. Ozboyaci, D. B. Kokh, S. Corni and R. C. Wade, Q. Rev. Biophys., 2016, 49.

81 B. S. Chhikara, B. Rathi and K. Parang, J. Mater. Nanosci., 2019, 6, 47-66.

82 E. A. Roman and F. L. G. Flecha, Biomolecules, 2014, 4, 354-373. 
E. Flapan, A. He and H. Wong, Proc. Natl. Acad. Sci. U. S. A., 2019, 116, 9360-9369.

84 walaa S. El-serwy, N. A. Mohamed, E. M. M. Kassem, E. S. Nossier and A. S. G. Shalaby, Chem. Biol. Lett., 2020, 7, 197-206.

H. Zare, A. Khodursky and V. Sartorelli, BMC Evol. Biol., , DOI:10.1186/1471-2148-14-74.

86 M. Wilkowska, W. Andrzejewska, R. Zieliński and M. Kozak, Biophys. J., 2018, 114, 221 a.

87 P. H. Nguyen, J. M. Campanera, S. T. Ngo, A. Loquet and P. Derreumaux, J. Phys. Chem. B, 2019, 123, 3643-3648.

88 A. Altis, P. H. Nguyen, R. Hegger and G. Stock, J. Chem. Phys., , DOI:10.1063/1.2746330.

89 S. J. Baumgart and B. Haendler, Int. J. Mol. Sci., 2017, 18.

90 I. E. Suka, N. F. Roslan, B. L. Chew, H. H. Goh, Z. Zainal and N. M. Isa, Sains Malaysiana, 2018, 47, 1465-1471.

91 D. H. Wolf and R. Menssen, FEBS Lett., 2018, 592, 2515-2524.

92 D. Bauer, S. Meinhold, R. P. Jakob, J. Stigler, U. Merkel, T. Maier, M. Rief and G. Žoldák, Proc. Natl. Acad. Sci. U. S. A., 2018, 115, 4666-4671.

93 A. Mashaghi, G. Kramer, P. Bechtluft, B. Zachmann-Brand, A. J. M. Driessen, B. Bukau and S. J. Tans, Nature, 2013, 500, 98-101. 